\title{
Stability properties of a crack inverse problem in half space
}

\author{
Darko Volkov and Yulong Jiang *
}

April 14, 2021

\begin{abstract}
We show in this paper a Lipschitz stability result for a crack inverse problem in half space. The direct problem is a Laplace equation with zero Neumann condition on the top boundary. The forcing term is a discontinuity across the crack. This formulation can be related to geological faults in elastic media or to irrotational incompressible flows in a half space minus an inner wall. The direct problem is well posed in an appropriate functional space. We study the related inverse problem where the jump across the crack is unknown, and more importantly, the geometry and the location of the crack are unknown. The data for the inverse problem is of Dirichlet type over a portion of the top boundary. We prove that this inverse problem is uniquely solvable under some assumptions on the geometry of the crack. The highlight of this paper is showing a stability result for this inverse problem. Assuming that the crack is planar, we show that reconstructing the plane containing the crack is Lipschitz stable despite the fact that the forcing term for the underlying PDE is unknown. This uniform stability result holds under the assumption that the forcing term is bounded above and the Dirichlet data is bounded below away from zero in appropriate norms.
\end{abstract}

\section{Introduction}

We consider a PDE model derived from a problem in geophysics where seismic or displacement data is collected by surface sensors and then processed to reconstruct a source and an unknown fault. Recently, there has been some progress in the understanding of the underlying mathematics. In particular, well posedness of the forward problem for the three dimensional linear elasticity model and uniqueness for the related inverse problem were shown in [12], and in [2, 1] where more general fault geometries and elasticity tensors were studied. A stability result in the case of planar faults was achieved in [11].

Here, we examine the case of a model involving the Laplace equation. This model is also relevant to geophysics: in dimension two, it relates to the so called anti-plane strain configuration. This configuration has already attracted much attention from geophysicists and

${ }^{*}$ Department of Mathematical Sciences, Worcester Polytechnic Institute, Worcester, MA 01609. Corresponding author email: darko@wpi.edu. 
mathematicians due to how simple and yet relevant the formulation is [9, 8, 4]. In dimension three, the model considered in this paper can be related to irrotational incompressible flows in a medium with a top wall and an inner wall.

We now outline the contents of this paper. In section 2, we first write out the governing partial differential equation for this problem for which we prove existence and uniqueness in an adequate functional space. This equation involves the Laplace operator for the unknown $u$ in the lower half space minus a crack $\Gamma$. Mathematically, $\Gamma$ is set to be an open Lipschitz surface. A zero Neumann condition is prescribed on the top boundary $x_{3}=0$ while the jump of $u$ across $\Gamma$ is set to be equal to some $g$. Next, we introduce the related crack inverse problem. For that problem, the additional knowledge of Dirichlet type data $\left.u\right|_{V}$ on a relatively open subset $V$ of the top boundary $x_{3}=0$ is provided. The challenge is that the geometry and the location of $\Gamma$ are unknown, as well as the jump $g$. Assuming that the crack $\Gamma$ is a bounded Lipschitz graph of a function in $\left(x_{1}, x_{2}\right)$ defined on a relatively open subset of the top boundary and one of the three conditions in Theorem 2.2 is satisfied, we are able to prove that the crack inverse problem is uniquely solvable. If all three conditions fail to be satisfied the crack inverse problem may not be uniquely solvable: we give an example in Appendix A.

Even in the simple case where $\Gamma$ is known, mapping $g$ to $\left.u\right|_{V}$ is a linear and compact operation, so its inverse is unbounded. To obtain a stability result, we assume that $\Gamma$ is included in the plane with equation $x_{3}=a x_{1}+b x_{2}+d$. The stability result, which is the highlight of this paper, is formulated in the geometry parameter $\boldsymbol{m}=(a, b, d)$. Theorem 3.3 , states that $\boldsymbol{m}$ depends on $\left.u\right|_{V}$ in a Lipschitz fashion for all jumps $g$ bounded above in $H_{0}^{1}$ and such that the corresponding $L^{2}$ norm of $\left.u\right|_{V}$ remains bounded below by a positive constant.

To put our work in perspective, let us first explain how our results compare to [6]. In that paper, it was shown in a two dimensional setting that applying two adequately chosen forcing terms on the boundary can uniquely determine cracks. The proof technique used in that paper is entirely different from the technique used here which is based on the inverse function theorem and forming a PDE on the surface $\Gamma$. The inverse problem considered in [6] is active, which means that boundary conditions can be adequately chosen for the result to hold. In contrast, we are interested in a passive inverse problem, so the forcing term $g$ is an unknown for the inverse problem. More recently, the authors of [2] studied a fault inverse problem closely related to the crack inverse problem considered here. That problem was formulated using the three dimensional elasticity equations and was analyzed from the viewpoint of very weak solutions. Unique-continuation arguments were used to establish uniqueness for the crack inverse problem. In [1] the case of layered media with piecewise Lipschitz Lamé coefficients was covered: uniqueness for the crack inverse problem was established under minimal assumptions for the fault geometry. In [11, a stability result closely related to Theorem 3.3 of this present paper was proved for the linear elasticity equation case. Due to the higher level of complexity of the elasticity equations, additional assumptions had to be made in order to obtain a stability result. The jump $g$ across $\Gamma$ in that case was modeled to be a tangential vector field. To obtain a stability estimate, it was assumed that $\Gamma$ is not horizontal, that $g$ is one-directional, or that $g$ is the gradient of a function in $H^{2}$. Theorem 4.1 in [1] provides a stability estimate which is uniform in all planar faults $\Gamma$ and jumps $g$, provided a geometry $\Gamma^{\prime}$ and a jump $g^{\prime}$ are fixed. In this paper, in Theorem 3.3, our uniform Lispchitz statement is stronger because it is also valid for all $\Gamma^{\prime}$ and all $g^{\prime}$ bounded above 
and below in appropriate norms.

\section{The direct and inverse crack problems in half space}

\subsection{Problem formulation and physical interpretation}

Let $\mathbb{R}^{3-}$ be the open half space $\left\{x_{3}<0\right\}$, where we use the notation $\boldsymbol{x}=\left(x_{1}, x_{2}, x_{3}\right)$ for vectors in $\mathbb{R}^{3}$. Let $\Gamma$ be a Lipschitz open surface in $\mathbb{R}^{3-}$ and $D$ a domain in $\mathbb{R}^{3-}$ with Lipschitz boundary such that $\Gamma \subset \partial D$. We assume that $\Gamma$ is strictly included in $\mathbb{R}^{3-}$ so that the distance from $\Gamma$ to the plane $\left\{x_{3}=0\right\}$ is positive. We define the direct crack problem to be the boundary value problem,

$$
\begin{array}{r}
\Delta u=0 \text { in } \mathbb{R}^{3-} \backslash \bar{\Gamma}, \\
\partial_{x_{3}} u=0 \text { on the surface } x_{3}=0, \\
{\left[\frac{\partial u}{\partial \boldsymbol{n}}\right]=0 \text { across } \Gamma,} \\
{[u]=g \text { across } \Gamma,} \\
u(\boldsymbol{x})=O\left(\frac{1}{|\boldsymbol{x}|}\right) \text { uniformly as }|\boldsymbol{x}| \rightarrow \infty,
\end{array}
$$

where $[v]$ denotes the jump of a function $v$ across $\Gamma$ in the normal direction, and $\boldsymbol{n}$ is a unit normal vector to $\Gamma$. In problem (145), $u$ can be a model for the potential of an irrotational flow, with an impermeable and immobile wall $\left\{x_{3}=0\right\}$. The flow is tangent to the inner wall $\Gamma$, and the discontinuity of the tangent flow is given by the tangential gradient of $g$. Alternatively, if we wrote the analog of problem (1,5) in two dimensions, it could model a strike-slip fault in geophysics where the equations of linear elasticity simplify to the scalar Laplacian. In that case the scalar function $u$ models displacements in the direction orthogonal to a cross-section, $g$ models a slip, and normal derivatives model traction [8, 9, 4,. A stability analysis for the inverse problem related to the two dimensional analog of (1, 5) will be the subject of another study.

\subsection{Existence and uniqueness for solutions to the direct problem}

We seek solutions to problem (1.5) that have local $H^{1}$ regularity. Due to the trace theorem (which is also valid in Lispchitz domains, [5, 7]), we require the forcing term $g$ to be in $\tilde{H}^{\frac{1}{2}}(\Gamma)$ which is the space of functions in $H^{\frac{1}{2}}(\partial D)$ supported in $\bar{\Gamma}$. As to $u$, we take it to be in the space

$$
\mathcal{V}=\left\{v \in H_{l o c}^{1}\left(\mathbb{R}^{3-} \backslash \bar{\Gamma}\right): \nabla v, \frac{v}{\sqrt{1+|\boldsymbol{x}|^{2}}} \in L^{2}\left(\mathbb{R}^{3-} \backslash \bar{\Gamma}\right)\right\}
$$

The natural norm $\|\nabla v\|_{L^{2}\left(\mathbb{R}^{3-} \backslash \bar{\Gamma}\right)}+\left\|\frac{v}{\sqrt{1+|\boldsymbol{x}|^{2}}}\right\|_{L^{2}\left(\mathbb{R}^{3-} \backslash \bar{\Gamma}\right)}$ on $\mathcal{V}$ is equivalent to the norm given by $\|\nabla v\|_{L^{2}\left(\mathbb{R}^{3-} \backslash \bar{\Gamma}\right)}$ according to Theorem A.1 in [15].

Theorem 2.1. Let $g$ be in $\tilde{H}^{1 / 2}(\Gamma)$. Problem (14) has a unique solution $u$ in $\mathcal{V}$. This solution satisfies the decay condition (5). 
Proof: We first show uniqueness. Assume that $g=0$. Let $v$ be a compactly supported element in $\mathcal{V}$ such that $[v]=0$ across $\Gamma$. By $(2), \int_{\left\{x_{3}=0\right\}}\left(\partial_{x_{3}} u\right) v=0$. Then, using Green's theorem and (1), (3), and (4), we find that $\int_{\mathbb{R}^{3}-} \nabla u \cdot \nabla v=0$. By a density argument, we then infer that $\int_{\mathbb{R}^{3}-}|\nabla u|^{2}=0$. But the definition of $\mathcal{V}$ precludes constant functions to be different from zero. Thus $u=0$ and uniqueness is shown.

Existence can be shown by setting up a variational problem for $u$ in $\mathcal{V}$. Instead, we choose to express $u$ as an integral over $\Gamma$ against an adequate Green function since this formulation will be crucial further in this study. Denote,

$$
\Phi(\boldsymbol{x}, \boldsymbol{y})=\frac{1}{4 \pi} \frac{1}{|\boldsymbol{x}-\boldsymbol{y}|},
$$

the free space Green function for the Laplacian. Then we can argue that

$$
u^{\prime}(\boldsymbol{x})=\int_{\Gamma} \nabla_{\boldsymbol{y}} \Phi(\boldsymbol{x}, \boldsymbol{y}) \cdot \boldsymbol{n}(\boldsymbol{y}) g(\boldsymbol{y}) d \sigma(\boldsymbol{y}),
$$

satisfies (1), (3), (4), (5), and is in $\mathcal{V}$. Indeed, as $g$ is in $\tilde{H}^{1 / 2}(\Gamma)$, we can extend $g$ by zero to a function in $H^{1 / 2}(\partial D)$ so $(7)$ equals

$$
u^{\prime}(\boldsymbol{x})=\int_{\partial D} \nabla_{\boldsymbol{y}} \Phi(\boldsymbol{x}, \boldsymbol{y}) \cdot \boldsymbol{n}(\boldsymbol{y}) g(\boldsymbol{y}) d \sigma(\boldsymbol{y}) .
$$

Even though $\partial D$ is only Lipschitz regular, by Theorem 1 in [3], $u^{\prime}$ is a function in $H_{\text {loc }}^{1}\left(\mathbb{R}^{3} \backslash \bar{\Gamma}\right)$ and by Lemma 4.1 in [3] the jump $\left[u^{\prime}\right]$ across $\Gamma$ is equal to $g$ almost everywhere, while the jump $\left[\frac{\partial u^{\prime}}{\partial \boldsymbol{n}}\right]$ is zero. To find a solution to the PDE 1,4 ) we then set

$$
u(\boldsymbol{x})=\int_{\Gamma} H(\boldsymbol{x}, \boldsymbol{y}) g(\boldsymbol{y}) d \sigma(\boldsymbol{y}),
$$

where

$$
H(\boldsymbol{x}, \boldsymbol{y})=\nabla_{\boldsymbol{y}} \Phi(\boldsymbol{x}, \boldsymbol{y}) \cdot \boldsymbol{n}(\boldsymbol{y})+\nabla_{\boldsymbol{y}} \Phi(\overline{\boldsymbol{x}}, \boldsymbol{y}) \cdot \boldsymbol{n}(\boldsymbol{y}),
$$

and $\overline{\boldsymbol{x}}=\left(x_{1}, x_{2},-x_{3}\right)$. Then conditions (1-4) are satisfied, $u(\boldsymbol{x})=O\left(\frac{1}{|\boldsymbol{x}|^{2}}\right)$, and $\nabla u(\boldsymbol{x})=$ $O\left(\frac{1}{|\boldsymbol{x}|^{3}}\right)$, uniformly in $\frac{\boldsymbol{x}}{|\boldsymbol{x}|}$, so $u$ is in $\mathcal{V}$.

\subsection{The crack inverse problem: formulation and uniqueness of solutions}

We now prove a theorem stating that the crack inverse problem related to problem (1) 5) has at most one solution. The data for the inverse problem is overdetermined boundary conditions over a portion of the top plane $\left\{x_{3}=0\right\}$. Both the discontinuity $g$ and the crack $\Gamma$ are unknown in the inverse problem. Our theorem is valid for surfaces $\Gamma$ that are graphs of functions $\psi: R \rightarrow \mathbb{R}$. We assume that $R$ is a bounded subset of $\mathbb{R}^{2}$ such that it is equal to the interior of its closure and that the closure of $\Gamma$ is in $\mathbb{R}^{3-}$. If we only assume that $\psi$ is Lipschitz regular, the crack inverse problem may not be uniquely solvable. We show a counter-example in Appendix A. 
Theorem 2.2. For $i=1,2$, let $R_{i}$ be an open and bounded subset of $\mathbb{R}^{2}$ such that $R_{i}$ is equal to the interior of its closure. Let $\Gamma_{i}$ be the surface defined by the graph of a Lipschitz function $\psi_{i}: R_{i} \rightarrow \mathbb{R}$. Assume that the closure of $\Gamma_{i}$ is in $\mathbb{R}^{3-}$. For $i=1,2$, let $u^{i}$ be the unique solution to (1.5) with $\Gamma_{i}$ in place of $\Gamma$ and the jumps $g^{i}$ in $\tilde{H}^{1 / 2}\left(\Gamma_{i}\right)$ in place of $g$ such that $g^{i}$ has full support in $\overline{\Gamma_{i}}$. Let $V$ be a non-empty relatively open subset of $\left\{x_{3}=0\right\}$. Assume that one of the three conditions hold,

1. $\psi_{i}$ is real analytic for $i=1,2$,

2. $g^{i}$ is not equal to a constant on any relatively open subset of $\Gamma_{i}$,

3. $\mathbb{R}^{3-} \backslash \overline{\Gamma_{1} \cup \Gamma_{2}}$ has only one connected component.

If $u^{1}=u^{2}$ on $V$, then $\Gamma_{1}=\Gamma_{2}$ and $g^{1}=g^{2}$ almost everywhere.

Remark: In practice, assumption 3 may be the most interesting. For example it is achieved if $\Gamma_{i}$ is planar as assumed further in this paper, or if it is the union of two polygons such as in the numerical reconstruction considered in [13].

Proof of Theorem 2.2 Let $U=\mathbb{R}^{3-} \backslash \overline{\Gamma_{1} \cup \Gamma_{2}}$ and set $u=u^{1}-u^{2}$ in $U$. Given that $\Gamma_{1}$ and $\Gamma_{2}$ are bounded, $U$ has only one unbounded connected component which will be denoted by $U_{1}$. Note that its boundary $\partial U_{1}$ contains the top boundary $\left\{x_{3}=0\right\}$ since $\Gamma_{1}$ and $\Gamma_{2}$ are bounded away from that plane. Now, $u$ satisfies $\Delta u=0$ in $U_{1}$ and $u=\partial_{x_{3}} u=0$ on $V$, so by the Cauchy Kowalevski theorem and analytic continuation, $u$ is zero in $U_{1}$. Let $U_{j}, j \neq 1$, be a bounded connected component of $U$. Note that $\partial U_{j}$ is not necessarily Lipschitz regular. However, $u$ is in $H^{1}\left(U_{j}\right)$ and satisfies $\Delta u=0$ in $U_{j}$ by construction. We show in Appendix $\mathrm{B}$ that although $\partial U_{j}$ may not be globally Lipschitz, it is the union of two Lipschitz pieces, defined by $x_{3}=\psi_{1}\left(x_{1}, x_{2}\right)$ and $x_{3}=\psi_{2}\left(x_{1}, x_{2}\right)$ where $\left(x_{1}, x_{2}\right)$ is in the closure of a bounded and connected open subset $\Omega_{j}$ of $\mathbb{R}^{2}$. We have $\psi_{1}\left(x_{1}, x_{2}\right)<\psi_{2}\left(x_{1}, x_{2}\right)$ for all $\left(x_{1}, x_{2}\right)$ in $\Omega_{j}$ or $\psi_{1}\left(x_{1}, x_{2}\right)>\psi_{2}\left(x_{1}, x_{2}\right)$ for all $\left(x_{1}, x_{2}\right)$ in $\Omega_{j}$ as shown in Appendix B, Lemma B.2, Extending $\psi_{1}$ and $\psi_{2}$ to Lipschitz continuous functions on $\overline{R_{1}}$ and $\overline{R_{2}}, \psi_{1}\left(x_{1}, x_{2}\right)=\psi_{2}\left(x_{1}, x_{2}\right)$, for $\left(x_{1}, x_{2}\right)$ on the boundary of $\Omega_{j}$ as shown in Appendix B, Lemma B.3. Due to relation (3), $\left[\frac{\partial u^{1}}{\partial \boldsymbol{n}}\right]$ is zero on the portion of $\partial U_{j}$ where $x_{3}=\psi_{1}\left(x_{1}, x_{2}\right)$. [ $\left.\frac{\partial u^{2}}{\partial \boldsymbol{n}}\right]$ is also zero on that portion by regularity, so $\left[\frac{\partial u}{\partial \boldsymbol{n}}\right]$ is zero. A similar argument shows that $\left[\frac{\partial u}{\partial \boldsymbol{n}}\right]$ is also zero on the portion of $\partial U_{j}$ where $x_{3}=\psi_{2}\left(x_{1}, x_{2}\right)$. As $u$ was proven to be zero in $U_{1}$ and the boundary of $U_{j}$ is included in the boundary of $U_{1}$, we find that $\frac{\partial u}{\partial \boldsymbol{n}}$ is zero on $\partial U_{j}$. Green's theorem can now be applied to $u$ in $U_{j}$ to find that $\nabla u=0$ in $U_{j}$. As $U_{j}$ is connected, $u$ is equal to some constant $C_{j}$ in $U_{j}$. If $j=1$, we set $C_{j}=0$.

Let $\left(y_{1}, y_{2}\right)$ be in $R_{1}$. Arguing by contradiction, assume that $\left(y_{1}, y_{2}\right) \notin \overline{R_{2}}$. Then there is an open ball $B\left(\left(y_{1}, y_{2}\right), \alpha\right)$ in $\mathbb{R}^{2}$ such that $B\left(\left(y_{1}, y_{2}\right), \alpha\right) \subset R_{1}$ and $B\left(\left(y_{1}, y_{2}\right), \alpha\right) \cap R_{2}=\emptyset$. Let $\boldsymbol{y}=\left(y_{1}, y_{2}, \psi_{1}\left(y_{1}, y_{2}\right)\right)$. Then there is an open ball $B(\boldsymbol{y}, \beta)$ in $\mathbb{R}^{3}$ such that $B(\boldsymbol{y}, \beta) \cap \Gamma_{2}=\emptyset$. Clearly $B(\boldsymbol{y}, \beta) \backslash \overline{\Gamma_{1}} \subset U_{1}$, thus $[u]=0$ in $B(\boldsymbol{y}, \beta) \cap \Gamma_{1}$. It is also clear that $\left[u^{2}\right]=0$ in $B(\boldsymbol{y}, \beta) \cap \Gamma_{1}$, so we infer that $\left[u^{1}\right]=0$ in $B(\boldsymbol{y}, \beta) \cap \Gamma_{1}$ : this contradicts the assumption that $\left[u^{1}\right]$ has full support in $\Gamma_{1}$. We have thus shown that $R_{1} \subset \overline{R_{2}}$. By switching the roles of $R_{1}$ and $R_{2}$, this shows that $\overline{R_{1}}=\overline{R_{2}}$. Due to our assumption on $R_{1}$ and $R_{2}$, this implies that $R_{1}=R_{2}$.

Now, let $R_{3}$ be the interior of the set $\left\{\left(x_{1}, x_{2}\right) \in R_{1}: \psi_{1}\left(x_{1}, x_{2}\right)=\psi_{2}\left(x_{1}, x_{2}\right)\right\}$. Since the halfline $\left\{\left(x_{1}, x_{2}, t \psi_{1}\left(x_{1}, x_{2}\right)\right): t \in(1, \infty)\right\}$ and the line segment $\left\{\left(x_{1}, x_{2}, t \psi_{1}\left(x_{1}, x_{2}\right)\right): t \in(0,1)\right\}$ 
are in $U_{1}$ for all $\left(x_{1}, x_{2}\right)$ in $R_{3}$, we can argue that $[u]=0$ in $R_{3} \times \psi_{1}\left(R_{3}\right)$, and thus $g^{1}=g^{2}$ in this set. In particular, if $\Gamma_{1}=\Gamma_{2}$ then $g^{1}=g^{2}$ almost everywhere.

There remains to show that $R_{3}=R_{1}$. Fix $\boldsymbol{y}=\left(y_{1}, y_{2}, \psi_{1}\left(y_{1}, y_{2}\right)\right)$ for $\left(y_{1}, y_{2}\right)$ in $R_{1}$. Arguing by contradiction, assume that $\psi_{1}\left(y_{1}, y_{2}\right) \neq \psi_{2}\left(y_{1}, y_{2}\right)$. Then, by continuity, $\psi_{1}\left(z_{1}, z_{2}\right) \neq$ $\psi_{2}\left(z_{1}, z_{2}\right)$, for all $\left(z_{1}, z_{2}\right)$ in an open neighborhood $W \subset R_{1}$ of $\left(y_{1}, y_{2}\right)$. Since $u^{2}$ is regular near $\boldsymbol{y}$, we can argue that $\left[u^{2}\left(y_{1}, y_{2}, \psi_{1}\left(y_{1}, y_{2}\right)\right)\right]=0$ for almost all $\left(y_{1}, y_{2}\right)$ in $W$, thus $\left[u^{1}\left(y_{1}, y_{2}, \psi_{1}\left(y_{1}, y_{2}\right)\right)\right]= \pm C_{j}$ for some $j$ and almost all $\left(y_{1}, y_{2}\right)$ in $W$. If condition 2 holds, this is a contradiction. If condition 3 holds, then $C_{j}=C_{1}=0$, and this contradicts that $g^{1}$ has full support in $\Gamma_{1}$. If condition 1 holds, given that there are no piecewise constant functions in $\tilde{H}^{\frac{1}{2}}\left(\Gamma_{i}\right)$ other than zero, if $C_{j} \neq 0$ for some $j$, there is an open set $S$ of $\mathbb{R}^{3-}$ such that $\Gamma_{1} \cap S \neq \emptyset$ and $\left[u^{1}\right]$ is not constant in $\Gamma_{1} \cap S$. But given what we showed earlier, we must have that $\Gamma_{1} \cap S=\Gamma_{2} \cap S$, that is, the functions $\psi_{1}$ and $\psi_{2}$ coincide on the open subset of $\mathbb{R}^{2}\left\{\left(x_{1}, x_{2}\right) \in \mathbb{R}^{2}:\left(x_{1}, x_{2}, x_{3}\right) \in \Gamma_{1} \cap S\right\}$. Thus if condition 1 holds, $\psi_{1}=\psi_{2}$ everywhere in $R_{1}=R_{2}$.

\subsection{High order jump formulas for derivatives of the double layer potential}

To prove stability results for the crack inverse problem, we will argue by contradiction. From the assumption that the crack inverse problem is not Lipschitz continuous, we will form a PDE on $\Gamma$ for the slip $g$, which we will show to only have the trivial solution, pointing to a contradiction. To form that PDE for $g$, we will need jump formulas for the integral expression (9) for $\boldsymbol{x}$ across $\Gamma$. Although the formula for the jump of $(9)$ across $\Gamma$ is well known, formulas for the jumps of its first and second derivatives are less known, so it is worth stating them in detail. A proof is provided in Appendix C.

With $\Phi$ as in (6) and $\boldsymbol{v}$ a vector in $\mathbb{R}^{3}$, we set for $\boldsymbol{x}, \boldsymbol{y}$ in $\mathbb{R}^{3-}$ such that $\boldsymbol{x} \neq \boldsymbol{y}$,

$$
\begin{array}{r}
G(\boldsymbol{x}, \boldsymbol{y}, \boldsymbol{v})=\nabla_{\boldsymbol{y}} \Phi(\boldsymbol{x}, \boldsymbol{y}) \cdot \boldsymbol{v} \\
H(\boldsymbol{x}, \boldsymbol{y}, \boldsymbol{v})=\nabla_{\boldsymbol{y}} \Phi(\boldsymbol{x}, \boldsymbol{y}) \cdot \boldsymbol{v},+\nabla_{\boldsymbol{y}} \Phi(\overline{\boldsymbol{x}}, \boldsymbol{y}) \cdot \boldsymbol{v}
\end{array}
$$

where as previously $\overline{\boldsymbol{x}}=\left(x_{1}, x_{2},-x_{3}\right)$.

For an open subset $V$ of $\mathbb{R}^{d}$, we denote $C_{c}^{\infty}(V)$ the space of smooth functions compactly supported in $V$. If $\Gamma$ is an open planar surface in $\mathbb{R}^{3}$, it can be mapped by rotation and translation to an open subset of $\mathbb{R}^{2}$ and this mapping can be used to define $C_{c}^{\infty}(\Gamma)$ by pullback.

Lemma 2.1. Let $\Gamma$ be a planar open surface in $\mathbb{R}^{3}$ with surface element $d S$, and unit normal vector $\boldsymbol{n}$. Let $\boldsymbol{t}$ be a fixed unit vector parallel to $\Gamma$. Let $g$ be in $L^{2}(\Gamma)$ and $\phi$ in $C_{c}^{\infty}(\Gamma)$. For $\epsilon>0, \boldsymbol{x}, \boldsymbol{y}$ in $\Gamma$, and $\boldsymbol{p}$ in $\mathbb{R}^{3}$ define the notation,

$$
G^{+-}(\boldsymbol{x}, \boldsymbol{y}, \boldsymbol{p})=G(\boldsymbol{x}+\epsilon \boldsymbol{n}, \boldsymbol{y}, \boldsymbol{p})-G(\boldsymbol{x}-\epsilon \boldsymbol{n}, \boldsymbol{y}, \boldsymbol{p}) .
$$

The following jump formulas across $\Gamma$ hold,

$$
\lim _{\epsilon \rightarrow 0} \int_{\Gamma} \int_{\Gamma} G^{+-}(\boldsymbol{x}, \boldsymbol{y}, \boldsymbol{n}) g(y) d S(\boldsymbol{y}) \phi(\boldsymbol{x}) d S(\boldsymbol{x})=\int_{\Gamma} g(\boldsymbol{x}) \phi(\boldsymbol{x}) d S(\boldsymbol{x}),
$$




$$
\begin{gathered}
\lim _{\epsilon \rightarrow 0} \int_{\Gamma} \int_{\Gamma} \frac{\partial_{\boldsymbol{y}} G^{+-}}{\partial \boldsymbol{t}}(\boldsymbol{x}, \boldsymbol{y}, \boldsymbol{n}) g(\boldsymbol{y}) d S(\boldsymbol{y}) \phi(\boldsymbol{x}) d S(\boldsymbol{x})=\int_{\Gamma} g(\boldsymbol{x}) \partial_{\boldsymbol{t}} \phi(\boldsymbol{x}) d S(\boldsymbol{x}), \\
\lim _{\epsilon \rightarrow 0} \int_{\Gamma} \int_{\Gamma} G^{+-}(\boldsymbol{x}, \boldsymbol{y}, \boldsymbol{t}) g(\boldsymbol{y}) d S(\boldsymbol{y}) \phi(\boldsymbol{x}) d S(\boldsymbol{x})=0 \\
\lim _{\epsilon \rightarrow 0} \int_{\Gamma} \int_{\Gamma} \frac{\partial_{\boldsymbol{y}} G^{+-}}{\partial \boldsymbol{n}}(\boldsymbol{x}, \boldsymbol{y}, \boldsymbol{n}) g(\boldsymbol{y}) d S(\boldsymbol{y}) \phi(\boldsymbol{x}) d S(\boldsymbol{x})=0 \\
\lim _{\epsilon \rightarrow 0} \int_{\Gamma}\left(\frac{\partial_{\boldsymbol{x}}}{\partial \boldsymbol{n}} \int_{\Gamma} G^{+-}(\boldsymbol{x}, \boldsymbol{y}, \boldsymbol{n}) g(\boldsymbol{y}) d S(\boldsymbol{y})\right) \phi(\boldsymbol{x}) d S(\boldsymbol{x})=0 \\
\lim _{\epsilon \rightarrow 0}\left(\frac{\partial_{\boldsymbol{x}}}{\partial \boldsymbol{n}} \int_{\Gamma} G^{+-}(\boldsymbol{x}, \boldsymbol{y}, \boldsymbol{t}) g(\boldsymbol{y}) d S(\boldsymbol{y})\right) \phi(\boldsymbol{x}) d S(\boldsymbol{x})=-\int_{\Gamma} g(\boldsymbol{x}) \partial_{\boldsymbol{t}} \phi(\boldsymbol{x}) d S(\boldsymbol{x}), \\
\lim _{\epsilon \rightarrow 0} \int_{\Gamma}\left(\frac{\partial_{\boldsymbol{x}}}{\partial \boldsymbol{n}} \int_{\Gamma} \frac{\partial_{\boldsymbol{y}} G^{+-}}{\partial \boldsymbol{n}}(\boldsymbol{x}, \boldsymbol{y}, \boldsymbol{n}) g(\boldsymbol{y}) d S(\boldsymbol{y})\right) \phi(\boldsymbol{x}) d S(\boldsymbol{x})=\int_{\Gamma} g(\boldsymbol{x}) \Delta_{\Gamma} \phi(\boldsymbol{x}) d S(\boldsymbol{x}) . \\
\lim _{\epsilon \rightarrow 0} \int_{\Gamma}\left(\frac{\partial_{\boldsymbol{x}}}{\partial \boldsymbol{n}} \int_{\Gamma} \frac{\partial_{\boldsymbol{y}} G^{+-}}{\partial \boldsymbol{t}}(\boldsymbol{x}, \boldsymbol{y}, \boldsymbol{n}) g(\boldsymbol{y}) d S(\boldsymbol{y})\right) \phi(\boldsymbol{x}) d S(\boldsymbol{x})=0 .
\end{gathered}
$$

\section{Lipschitz stability results for the half space crack inverse problem in case of planar geometries}

For a bounded relatively open set $R$ in the plane $\left\{x_{3}=0\right\}$ and $\boldsymbol{m}=(a, b, d)$ in $\mathbb{R}^{3}$, we define the surface

$$
\Gamma_{\boldsymbol{m}}=\left\{\left(x_{1}, x_{2}, a x_{1}+b x_{2}+d\right):\left(x_{1}, x_{2}\right) \in R\right\} .
$$

Let $B$ be a set of $\boldsymbol{m}$ in $\mathbb{R}^{3}$ such that $\Gamma_{\boldsymbol{m}} \subset \mathbb{R}^{3-}$. We assume that $B$ is closed and bounded. It follows that there is a positive constant $\beta$ such that

$$
\operatorname{dist}\left(\Gamma_{\boldsymbol{m}},\left\{x_{3}=0\right\}\right) \geq \beta,
$$

for all $\boldsymbol{m}$ in $B$, where dist is the distance between sets. We choose the unit normal vector on $\Gamma_{\boldsymbol{m}}$ to be $\boldsymbol{n}=\frac{(-a,-b, 1)}{\sqrt{a^{2}+b^{2}+1}}$. The surface element on $\Gamma_{\boldsymbol{m}}$ will be denoted by $\sigma d x_{1} d x_{2}=$ $\sqrt{a^{2}+b^{2}+1} d x_{1} d x_{2}$. Using (9) and (12), we introduce the linear compact operator mapping jumps $g$ across $\Gamma_{\boldsymbol{m}}$ to Dirichlet data on $V$,

$$
\begin{array}{r}
A_{\boldsymbol{m}}: H_{0}^{1}(R) \rightarrow L^{2}(V), \\
\left(A_{\boldsymbol{m}} g\right)(\boldsymbol{x})=\int_{R} H\left(x_{1}, x_{2}, 0, y_{1}, y_{2}, a y_{1}+b y_{2}+d, \boldsymbol{n}\right) g\left(y_{1}, y_{2}\right) \sigma d y_{1} d y_{2},
\end{array}
$$

where $\boldsymbol{x}=\left(x_{1}, x_{2}, 0\right)$ is in $V$. 
We know from Theorem 2.2 that $A_{\boldsymbol{m}}$ is injective. Fix a non-zero $h$ in $H_{0}^{1}(R)$ and define the function $\phi: B \rightarrow L^{2}(V)$ by

$$
\phi(\boldsymbol{m})=A_{\boldsymbol{m}} h .
$$

Due to the regularity of the Green's function $H$ and (21), we know that $\phi$ is analytic in $\boldsymbol{m}$. Theorem 2.2 implies that $\phi$ is injective. We will show that the inverse of $\phi$ defined on $\phi(B)$ and valued in $B$ is of class $C^{1}$ by applying the inverse function Theorem. As a consequence, $\phi^{-1}$ is Lipschitz continuous, which will yield our first stability estimate.

Theorem 3.1. Fix a non-zero $h$ in $H_{0}^{1}(R)$ and define the function $\phi$ from $B$ to $L^{2}(V)$ by (23). Then there is a positive constant $C$ such that

$$
C\left|\boldsymbol{m}-\boldsymbol{m}^{\prime}\right| \leq\left\|\phi(\boldsymbol{m})-\phi\left(\boldsymbol{m}^{\prime}\right)\right\|_{L^{2}(V)}
$$

for all $\boldsymbol{m}$ and $\boldsymbol{m}^{\prime}$ in $B$.

Proof: Let $\mathcal{B}$ be an open set of $\mathbb{R}^{3}$ such that $B \subset \mathcal{B}$ and for all $\boldsymbol{m}$ in $\mathcal{B}$

$$
\operatorname{dist}\left(\Gamma_{\boldsymbol{m}},\left\{x_{3}=0\right\}\right)>\frac{\beta}{2},
$$

where $\beta$ is as in (21). Arguing by contradiction, suppose that there is an $\boldsymbol{m}$ in $\mathcal{B}$ such that $\nabla \phi(\boldsymbol{m})$ does not have full rank. Then there is a non-zero vector $\left(\gamma_{1}, \gamma_{2}, \gamma_{3}\right)$ such that,

$$
\gamma_{1} \frac{\partial}{\partial a} \phi(\boldsymbol{m})+\gamma_{2} \frac{\partial}{\partial b} \phi(\boldsymbol{m})+\gamma_{3} \frac{\partial}{\partial d} \phi(\boldsymbol{m})=0 .
$$

We note that $\boldsymbol{n} \sigma$ simplifies to $(-a,-b, 1)$. Since $H(\boldsymbol{x}, \boldsymbol{y}, \boldsymbol{n})$ is linear in $\boldsymbol{n}$, we can apply the chain rule with $y_{3}=a y_{1}+b y_{2}+d$ on $\Gamma_{\boldsymbol{m}}$ to find that

$$
\begin{aligned}
\frac{\partial}{\partial a} H(\boldsymbol{x}, \boldsymbol{y}, \boldsymbol{n} \sigma) & =\frac{\partial y_{3}}{\partial a}\left(\partial_{y_{3}} H\right)(\boldsymbol{x}, \boldsymbol{y}, \boldsymbol{n} \sigma)+H\left(\boldsymbol{x}, \boldsymbol{y}, \frac{\partial(\boldsymbol{n} \sigma)}{\partial a}\right) \\
& =y_{1}\left(\partial_{y_{3}} H\right)(\boldsymbol{x}, \boldsymbol{y}, \boldsymbol{n} \sigma)-H\left(\boldsymbol{x}, \boldsymbol{y}, \boldsymbol{e}_{1}\right) .
\end{aligned}
$$

Similarly,

$$
\frac{\partial}{\partial b} H(\boldsymbol{x}, \boldsymbol{y}, \boldsymbol{n} \sigma)=y_{2}\left(\partial_{y_{3}} H\right)(\boldsymbol{x}, \boldsymbol{y}, \boldsymbol{n} \sigma)-H\left(\boldsymbol{x}, \boldsymbol{y}, \boldsymbol{e}_{2}\right)
$$

and

$$
\frac{\partial}{\partial d} H(\boldsymbol{x}, \boldsymbol{y}, \boldsymbol{n} \sigma)=\left(\partial_{y_{3}} H\right)(\boldsymbol{x}, \boldsymbol{y}, \boldsymbol{n} \sigma)
$$

For $\left(y_{1}, y_{2}\right)$ in $R$, define $f\left(y_{1}, y_{2}\right)=\gamma_{1} y_{1}+\gamma_{2} y_{2}+\gamma_{3}$. Since $h$ is independent of $a, b$, and $d$, we substitute 27,29 into 26 to find that

$$
\begin{gathered}
\int_{R}\left(\partial_{y_{3}} H\right)\left(\boldsymbol{x}, y_{1}, y_{2}, a y_{1}+b y_{2}+d, \boldsymbol{n}\right) h\left(y_{1}, y_{2}\right) f\left(y_{1}, y_{2}\right) \sigma d y_{1} d y_{2} \\
-\int_{R} H\left(\boldsymbol{x}, y_{1}, y_{2}, a y_{1}+b y_{2}+d, \nabla f\right) h\left(y_{1}, y_{2}\right) d y_{1} d y_{2}=0
\end{gathered}
$$


for all $\boldsymbol{x}$ in $V$. Set $w(\boldsymbol{x})$ to be the left hand side of $(30)$ where $\boldsymbol{x}$ has been extended to $\mathbb{R}^{3-} \backslash \Gamma_{\boldsymbol{m}}$. We will now show that $w$ is zero in $\mathbb{R}^{3-} \backslash \Gamma_{\boldsymbol{m}}$. Since $\partial_{y_{3}}$ and $\partial_{x_{i}}$ commute, we know from the definition of the Green's function that $w$ satisfies the Laplace Equation in $\mathbb{R}^{3-} \backslash \Gamma_{\boldsymbol{m}}$. We also know that $w$ is 0 on $V$ thanks to (30). By construction of the Green's tensor $H$, we know that for any $x$ on the plane $x_{3}=0$, any $\boldsymbol{y} \in \mathbb{R}^{3-}$ and any fixed vector $\boldsymbol{p} \in \mathbb{R}^{3}$, using the notation 12 ,

$$
\partial_{x_{3}} H(\boldsymbol{x}, \boldsymbol{y}, \boldsymbol{p})=0 .
$$

Thus we can take a $\partial_{y_{3}}$ derivative and commute it with $\partial_{x_{3}}$ to obtain

$$
\partial_{x_{3}} \partial_{y_{3}} H(\boldsymbol{x}, \boldsymbol{y}, \boldsymbol{p})=0 .
$$

It follows that $\partial_{x_{3}} w$ is also zero in $V$. As $w$ is zero in $V$, and $\Delta w=0$ in $\mathbb{R}^{3-} \backslash \overline{\Gamma_{m}}, w=0$ everywhere in $\mathbb{R}^{3-} \backslash \Gamma_{m}$ by the Cauchy Kowalevski theorem. In particular, the jump of $w$ across $\Gamma_{m}$ must be zero. As mentioned earlier, $H(\boldsymbol{x}, \boldsymbol{y}, \boldsymbol{p})-G(\boldsymbol{x}, \boldsymbol{y}, \boldsymbol{p})$ is smooth for any $\boldsymbol{x}, \boldsymbol{y}$ in $\mathbb{R}^{3-}$ and any fixed vector $\boldsymbol{p}$ in $\mathbb{R}^{3}$, therefore the jump across $\Gamma_{\boldsymbol{m}}$ of

$$
\begin{array}{r}
\int_{R}\left(\partial_{y_{3}} G\right)\left(\boldsymbol{x}, y_{1}, y_{2}, a y_{1}+b y_{2}+d, \boldsymbol{n}\right) h\left(y_{1}, y_{2}\right) f\left(y_{1}, y_{2}\right) \sigma d y_{1} d y_{2} \\
-\int_{R} G\left(x, y_{1}, y_{2}, a y_{1}+b y_{2}+d, \nabla f\right) h\left(y_{1}, y_{2}\right) d y_{1} d y_{2}
\end{array}
$$

is also zero. We now consider two cases.

Case 1: $(a, b) \neq 0$.

In this case the fault $\Gamma_{m}$ is not horizontal. Let $\alpha$ and $\beta$ be in $\mathbb{R}$ and $\boldsymbol{t}$ a unit vector in $\mathbb{R}^{3}$ parallel to to $\Gamma_{m}$ such that,

$$
\boldsymbol{e}_{3}=\alpha \boldsymbol{n}+\beta \boldsymbol{t}
$$

Then 31 can be rewritten as

$$
\begin{aligned}
\alpha \int_{R} & \frac{\partial_{\boldsymbol{y}} G}{\partial \boldsymbol{n}}\left(\boldsymbol{x}, y_{1}, y_{2}, a y_{1}+b y_{2}+d, \boldsymbol{n}\right) h\left(y_{1}, y_{2}\right) f\left(y_{1}, y_{2}\right) \sigma d y_{1} d y_{2} \\
& +\beta \int_{R} \frac{\partial_{\boldsymbol{y}} G}{\partial \boldsymbol{t}}\left(\boldsymbol{x}, y_{1}, y_{2}, a y_{1}+b y_{2}+d, \boldsymbol{n}\right) h\left(y_{1}, y_{2}\right) f\left(y_{1}, y_{2}\right) \sigma d y_{1} d y_{2} \\
& -\int_{R} G\left(\boldsymbol{x}, y_{1}, y_{2}, a y_{1}+b y_{2}+d, \nabla f\right) h\left(y_{1}, y_{2}\right) d y_{1} d y_{2}
\end{aligned}
$$

which is again zero for $\boldsymbol{x}$ in $\mathbb{R}^{3-} \backslash \overline{\Gamma_{\boldsymbol{m}}}$. We now apply lemma 2.1 to be more precise, respectively formulas (13) to 16 to obtain the weak equation

$$
-\beta \frac{\partial}{\partial \boldsymbol{t}}(h f)-\frac{1}{\sigma}(\nabla f \cdot \boldsymbol{n}) h=0 .
$$

Since in case $1(a, b) \neq 0, \boldsymbol{e}_{3} \neq \boldsymbol{n}$, so we infer that $\beta \neq 0$. As $h$ is in $H_{0}^{1}(R)$, equation (34) implies that $h$ is zero: this is due to lemma 3.3 in [11]. Thus we have contradicted the assumption $h \neq 0$. 
Case 2: $a$ and $b$ are zero.

In that case the fault $\Gamma_{\boldsymbol{m}}$ is horizontal. Recalling that $w(\boldsymbol{x})=0$ for all $x$ in $\mathbb{R}^{3-} \backslash \overline{\Gamma_{\boldsymbol{m}}}$, its $x_{3}$ derivative is also zero in the same open set thus the jump across $\Gamma_{m}$ of

$$
\begin{array}{r}
\partial_{x_{3}} w(\boldsymbol{x})=\partial_{x_{3}} \int_{R}\left(\partial_{y_{3}} G\right)\left(\boldsymbol{x}, y_{1}, y_{2}, d, \boldsymbol{e}_{3}\right) h\left(y_{1}, y_{2}\right) f\left(y_{1}, y_{2}\right) d y_{1} d y_{2} \\
-\partial_{x_{3}} \int_{R} G\left(\boldsymbol{x}, y_{1}, y_{2}, d, \nabla f\right) h\left(y_{1}, y_{2}\right) d y_{1} d y_{2}
\end{array}
$$

is zero. We then apply the jump formulas (69) 71) from Lemma C.2 to obtain the weak equation, $\Delta(f h)-\nabla f \cdot \nabla h=0$. Since $\Delta f=0$, this simplifies to $\operatorname{div}(f \nabla h)=0$, in other words

$$
\int_{R} f \nabla h \cdot \nabla \varphi=0
$$

for all $\varphi$ in $H_{0}^{1}(R)$. Recall that $h$ is in $H_{0}^{1}(R)$ and $f\left(x_{1}, x_{2}\right)=\gamma_{1} x_{1}+\gamma_{2} x_{2}+\gamma_{3}$ with $\left(\gamma_{1}, \gamma_{2}, \gamma_{3}\right) \neq 0$. If $f$ does not change signs in $R$, (36) clearly implies that $h$ is zero. If $f$ changes signs in $R$, then there is an open set $R^{+}$in $R$ where $f>0$, and an open set $R^{-}$ where $f<0 . R^{+}$and $R^{-}$are separated by a line. Without loss of generality, we can change coordinate systems in $\mathbb{R}^{2}$ by rotation and translation so that this line becomes the line with equation $x_{1}=0$ and we can rescale $f$ so that it becomes $f\left(x_{1}, x_{2}\right)=x_{1} . R^{+}$is then the set $\left\{\left(x_{1}, x_{2}\right) \in R: x_{1}>0\right\}$. For $\epsilon>0$, let $p_{\epsilon}$ be the function from $\mathbb{R}^{2}$ to $[0,1]$ be defined by

$$
p_{\epsilon}\left(x_{1}, x_{2}\right)= \begin{cases}1 & \text { if } x_{1} \geq \epsilon \\ \frac{x_{1}}{\epsilon} & \text { if } 0 \leq x_{1}<\epsilon, \\ 0 & \text { if } x_{1}<0\end{cases}
$$

$p_{\epsilon}$ is Lipschitz continuous and $p_{\epsilon} h$ is in $H_{0}^{1}(R)$. By (36), $\int_{R} f \nabla h \cdot \nabla\left(p_{\epsilon} h\right)=0$, which we write as

$$
\int_{R} p_{\epsilon} f|\nabla h|^{2}+\int_{R} h \nabla h \cdot f \nabla p_{\epsilon}=0 .
$$

As $\epsilon$ tends to zero the limit of the first term in (37) is $\int_{R} f^{+}|\nabla h|^{2}$, where $f^{+}$is the positive part of $f$. To tackle the second term, we note that $h$ is also in $L^{3}(R)$ by the Sobolev embedding theorem, thus by the generalized Hölder's inequality, $h \nabla h$ is in $L^{\frac{6}{5}}(R)$, as $\frac{1}{2}+\frac{1}{3}=\frac{5}{6}$. It follows, using Hölder's inequality, that

$$
\left|\int_{R} h \nabla h \cdot f \nabla p_{\epsilon}\right| \leq\|h \nabla h\|_{L^{\frac{6}{5}(R)}}\left(\int_{R}\left|f \nabla p_{\epsilon}\right|^{6}\right)^{\frac{1}{6}} .
$$

But now

$$
\int_{R}\left|f \nabla p_{\epsilon}\right|^{6}=\int_{R \cap\left\{\left(x_{1}, x_{2}\right): 0<x_{1}<\epsilon\right\}}\left(\frac{x_{1}}{\epsilon}\right)^{6} d x_{1} d x_{2}
$$

is $O(\epsilon)$ thanks to the rescaling $y_{1}=\frac{x_{1}}{\epsilon}$, thus we have shown that the second term in (37) converges to zero, thus $\int_{R} f^{+}|\nabla h|^{2}=0$. Similarly, we can show that $\int_{R} f^{-}|\nabla h|^{2}=0$, so 
altogether, $\int_{R}|f||\nabla h|^{2}=0$. As $f$ is a non-zero affine function, this implies that $h$ is zero almost everywhere: contradiction.

In summary, we have proved that $\nabla \phi(\boldsymbol{m})$ has full rank for any $\boldsymbol{m}$ in $\mathcal{B}$. The inverse function theorem guarantees that $\phi$ defines a $C^{1}$ diffeomorphism from an open neighborhood $U_{m}$ of $\boldsymbol{m}$ in $\mathcal{B}$ to its image by $\phi$ in $L^{2}(V)$. Arguing by contradiction, assume that estimate (24) does not hold. Then there are two sequences $\boldsymbol{p}_{n}$ and $\boldsymbol{q}_{n}$ in $B$ such that $\boldsymbol{p}_{n} \neq \boldsymbol{q}_{n}$ and

$$
\lim _{n \rightarrow \infty} \frac{\left\|\phi\left(\boldsymbol{p}_{n}\right)-\phi\left(\boldsymbol{q}_{n}\right)\right\|_{L^{2}(V)}}{\left|\boldsymbol{p}_{n}-\boldsymbol{q}_{n}\right|}=0 .
$$

As $B$ is compact, without loss of generality we may assume that $\boldsymbol{p}_{n}$ converges to some $\tilde{\boldsymbol{m}}$ in $B$ and $\boldsymbol{q}_{n}$ converges to some $\tilde{\tilde{\boldsymbol{m}}}$ in $B$. If $\tilde{\boldsymbol{m}} \neq \tilde{\tilde{\boldsymbol{m}}}$, this contradicts the uniqueness Theorem 2.2 , so we claim that both $\boldsymbol{p}_{n}$ and $\boldsymbol{q}_{n}$ converge to $\tilde{\boldsymbol{m}}$ in $B$. But we proved that $\phi$ defines a $C^{1}$ diffeomorphism in a open neighborhood $U_{\tilde{\boldsymbol{m}}}$ of $\tilde{\boldsymbol{m}}$, thus there exists a positive constant $C_{\tilde{\boldsymbol{m}}}$ such that for all $n$ large enough

$$
\left|\boldsymbol{p}_{n}-\boldsymbol{q}_{n}\right| \leq C_{\tilde{\boldsymbol{m}}}\left\|\phi\left(\boldsymbol{p}_{n}\right)-\phi\left(\boldsymbol{q}_{n}\right)\right\|_{L^{2}(V)},
$$

which contradicts $(38)$.

Stability Theorem 3.1 holds for a fixed jump $h$ in $H_{0}^{1}(R)$, but jumps are unknown in the crack inverse problem. The following result addresses this important issue. A Lipschitz stability statement in $h$ is not possible due the compactness of the operator $A_{m}$, however even if the jump $h$ is variable, a Lipschitz stability result in the geometry parameter $\boldsymbol{m}$ holds as shown below.

Theorem 3.2. Let $B$ be a closed and bounded subset of geometry parameters $\boldsymbol{m}$ in $\mathbb{R}^{3}$ such that the distance condition (21) holds. Let $A_{m}$ be the crack to boundary operator defined by (22). Fix a non-zero $h_{0}$ in $H_{0}^{1}(R)$ and $\boldsymbol{m}_{0}$ in $B$. There exists a positive constant $C$ such that

$$
\inf _{h \in H_{0}^{1}(R)}\left\|A_{\boldsymbol{m}} h-A_{\boldsymbol{m}_{0}} h_{0}\right\|_{L^{2}(V)} \geq C\left|\boldsymbol{m}-\boldsymbol{m}_{0}\right|
$$

for all $m$ in $B$.

Proof: Let $P_{m}$ be the orthogonal projection onto $\overline{\mathcal{R}\left(A_{m}\right)}$, the closure of the range of $A_{m}$ in $L^{2}(V)$ for $\boldsymbol{m}$ in $B$. We first claim that,

$$
\left\|P_{\boldsymbol{m}}-P_{\boldsymbol{m}_{0}}\right\|=O\left(\left|\boldsymbol{m}-\boldsymbol{m}_{0}\right|\right) .
$$

Indeed, since the nullspaces of $A_{m}^{*}$ and $A_{m} A_{m}^{*}$ are equal, taking their orthogonal we find that $\overline{\mathcal{R}\left(A_{m}\right)}=\overline{\mathcal{R}\left(A_{m} A_{m}^{*}\right)}$. The orthogonal projection on $\overline{\mathcal{R}\left(A_{m} A_{m}^{*}\right)}$ can be expressed by the contour integral [10]

$$
P_{m}=\frac{1}{2 i \pi} \int_{\mathcal{C}}\left(\zeta I-A_{m} A_{m}^{*}\right)^{-1} d \zeta
$$

where $\mathcal{C}$ is the circle in the complex plane centered at the origin with radius $\left\|A_{\boldsymbol{m}} A_{\boldsymbol{m}}^{*}\right\|+1$. Since $\left\|A_{\boldsymbol{m}} A_{\boldsymbol{m}}^{*}-A_{\boldsymbol{m}_{0}} A_{\boldsymbol{m}_{0}}^{*}\right\|=O\left(\left|\boldsymbol{m}-\boldsymbol{m}_{0}\right|\right)$, estimate (40) holds. 
Arguing by contradiction, assume that there is a sequence $\boldsymbol{m}_{n}$ in $B$ and a sequence $h_{n}$ in $H_{0}^{1}(R)$ such that $\boldsymbol{m}_{n} \neq \boldsymbol{m}_{0}$ for all $n \geq 1$ and

$$
\left\|A_{\boldsymbol{m}_{0}} h_{0}-A_{\boldsymbol{m}_{n}} h_{m_{n}}\right\|_{L^{2}(V)}=o\left(\left|\boldsymbol{m}_{n}-\boldsymbol{m}_{0}\right|\right) .
$$

Since $I-P_{\boldsymbol{m}_{n}}$ is an orthogonal projection,

$$
\left\|A_{\boldsymbol{m}_{0}} h_{0}-A_{\boldsymbol{m}_{n}} h_{\boldsymbol{m}_{n}}\right\|_{L^{2}(V)} \geq\left\|\left(I-P_{\boldsymbol{m}_{n}}\right)\left(A_{\boldsymbol{m}_{0}} h_{0}-A_{\boldsymbol{m}_{n}} h_{\boldsymbol{m}_{n}}\right)\right\|_{L^{2}(V)},
$$

thus by 41,

$$
\left\|\left(I-P_{\boldsymbol{m}_{n}}\right) A_{\boldsymbol{m}_{0}} h_{0}\right\|_{L^{2}(V)}=o\left(\left|\boldsymbol{m}_{n}-\boldsymbol{m}_{0}\right|\right),
$$

or equivalently,

$$
\left\|\left(I-P_{\boldsymbol{m}_{n}}\right)\left(A_{\boldsymbol{m}_{n}}-A_{\boldsymbol{m}_{0}}\right) h_{0}\right\|_{L^{2}(V)}=o\left(\left|\boldsymbol{m}_{n}-\boldsymbol{m}_{0}\right|\right) .
$$

By 40$)$,

$$
\left\|\left(P_{\boldsymbol{m}_{0}}-P_{\boldsymbol{m}_{n}}\right)\left(A_{\boldsymbol{m}_{n}}-A_{\boldsymbol{m}_{0}}\right) h_{0}\right\|_{L^{2}(V)}=o\left(\left|\boldsymbol{m}_{n}-\boldsymbol{m}_{0}\right|\right),
$$

thus combining (42) and 43), we find,

$$
\left\|\left(I-P_{\boldsymbol{m}_{0}}\right)\left(A_{\boldsymbol{m}_{n}}-A_{\boldsymbol{m}_{0}}\right) h_{0}\right\|_{L^{2}(V)}=o\left(\left|\boldsymbol{m}_{n}-\boldsymbol{m}_{0}\right|\right) .
$$

Equivalently,

$$
\left(I-P_{\boldsymbol{m}_{0}}\right) \frac{\left(A_{\boldsymbol{m}_{n}}-A_{\boldsymbol{m}_{0}}\right)}{\left|\boldsymbol{m}_{n}-\boldsymbol{m}_{0}\right|} h_{0}=o(1) .
$$

As $\frac{\boldsymbol{m}_{n}-\boldsymbol{m}_{0}}{\left|\boldsymbol{m}_{n}-\boldsymbol{m}_{0}\right|}$ is a sequence on the unit sphere of $\mathbb{R}^{3}$, after possibly extracting a subsequence we may assume that it converges to some $\boldsymbol{q}$ with $|\boldsymbol{q}|=1$. To keep notations consistent with those used in the proof of Theorem (3.1), we set $q=\left(\gamma_{1}, \gamma_{2}, \gamma_{3}\right), \boldsymbol{m}_{0}=(a, b, d)$, and $f\left(y_{1}, y_{2}\right)=\gamma_{1} y_{1}+\gamma_{2} y_{2}+\gamma_{3}$. We define the linear operator

$$
\begin{array}{r}
\partial_{\boldsymbol{q}} A_{\boldsymbol{m}_{0}}: H_{0}^{1}(R) \rightarrow L^{2}(V) \\
\partial_{\boldsymbol{q}} A_{\boldsymbol{m}_{0}}=\gamma_{1} \frac{\partial}{\partial a} A_{\boldsymbol{m}_{0}}+\gamma_{2} \frac{\partial}{\partial b} A_{\boldsymbol{m}_{0}}+\gamma_{3} \frac{\partial}{\partial d} A_{\boldsymbol{m}_{0}} .
\end{array}
$$

Taking the limit as $n \rightarrow \infty$ in 45) we find,

$$
\left(I-P_{\boldsymbol{m}_{0}}\right) \partial_{\boldsymbol{q}} A_{\boldsymbol{m}_{0}} h_{0}=0,
$$

thus, there is a $g_{0}$ in $H_{0}^{1}(R)$ such that,

$$
\partial_{\boldsymbol{q}} A_{m_{0}} h_{0}-A_{m_{0}} g_{0}=0 .
$$


We now show that (48) implies that $h_{0}=0$, which is a contradiction. Based on (48), we define

$$
\begin{aligned}
w^{\prime}(\boldsymbol{x})= & \int_{R}\left(\partial_{y_{3}} H\right)\left(\boldsymbol{x}, y_{1}, y_{2}, a y_{1}+b y_{2}+d, \boldsymbol{n}\right) h_{0}\left(y_{1}, y_{2}\right) f\left(y_{1}, y_{2}\right) \sigma d y_{1} d y_{2} \\
& -\int_{R} H\left(\boldsymbol{x}, y_{1}, y_{2}, a y_{1}+b y_{2}+d, \nabla f\right) h_{0}\left(y_{1}, y_{2}\right) d y_{1} d y_{2} \\
& -\int_{R} H\left(\boldsymbol{x}, y_{1}, y_{2}, a y_{1}+b y_{2}+d, \boldsymbol{n}\right) g_{0}\left(y_{1}, y_{2}\right) \sigma d y_{1} d y_{2},
\end{aligned}
$$

for $\boldsymbol{x}$ in $\mathbb{R}^{3-} \backslash \overline{\Gamma_{\boldsymbol{m}_{0}}}, w^{\prime}(\boldsymbol{x})$ is zero for $\boldsymbol{x}$ in $V$ because of relation $(48)$. As $\partial_{x_{3}} w^{\prime}(\boldsymbol{x})$ is also zero for $\boldsymbol{x}$ in $V$, by the same argument as in the proof of Theorem 3.1, we infer that $w^{\prime}(\boldsymbol{x})=0$ for all $\boldsymbol{x}$ in $\mathbb{R}^{3-} \backslash \overline{\Gamma_{\boldsymbol{m}_{0}}}$. Now, unlike, in the proof of Theorem 3.1, we have to contend with the inhomogeneity presented by the $g_{0}$ term in 490 . To eliminate that term, we take the jump $\left[\partial_{\boldsymbol{n}} w^{\prime}\right]$ across $\Gamma_{\boldsymbol{m}_{0}}$. Once again we use that $H-G$ is smooth in $\mathbb{R}^{3-} \times \mathbb{R}^{3-}$, thus we only need to consider the jump of the normal derivative across $\Gamma_{\boldsymbol{m}_{0}}$ of

$$
\begin{array}{r}
\alpha \int_{R} \frac{\partial_{\boldsymbol{y}} G}{\partial \boldsymbol{n}}\left(\boldsymbol{x}, y_{1}, y_{2}, a y_{1}+b y_{2}+d, \boldsymbol{n}\right) h_{0}\left(y_{1}, y_{2}\right) f\left(y_{1}, y_{2}\right) \sigma d y_{1} d y_{2} \\
+\beta \int_{R} \frac{\partial_{\boldsymbol{y}} G}{\partial \boldsymbol{t}}\left(\boldsymbol{x}, y_{1}, y_{2}, a y_{1}+b y_{2}+d, \boldsymbol{n}\right) h_{0}\left(y_{1}, y_{2}\right) f\left(y_{1}, y_{2}\right) \sigma d y_{1} d y_{2} \\
\quad-\int_{R} G\left(\boldsymbol{x}, y_{1}, y_{2}, a y_{1}+b y_{2}+d, \nabla f\right) h_{0}\left(y_{1}, y_{2}\right) d y_{1} d y_{2} \\
-\int_{R} G\left(\boldsymbol{x}, y_{1}, y_{2}, a y_{1}+b y_{2}+d, \boldsymbol{n}\right) g_{0}\left(y_{1}, y_{2}\right) \sigma d y_{1} d y_{2}
\end{array}
$$

where $\alpha, \beta, \boldsymbol{t}$ are as in (32). To compute this jump, we respectively apply formulas (19), (20), 117) and (18), and (17). Doing so, we obtain the weak equation on $\Gamma_{\boldsymbol{m}_{0}}$,

$$
\alpha \Delta_{\Gamma_{\boldsymbol{m}_{0}}}\left(\tilde{f} \tilde{h_{0}}\right)-\frac{1}{\sigma} \nabla_{\Gamma_{\boldsymbol{m}_{0}}} \tilde{f} \cdot \nabla_{\Gamma_{\boldsymbol{m}_{0}}} \tilde{h_{0}}=0,
$$

where $\tilde{h_{0}}$ is the function in $H_{0}^{1}\left(\Gamma_{\boldsymbol{m}_{0}}\right)$ defined by $\tilde{h_{0}}\left(x_{1}, x_{2}, a x_{1}+b x_{2}+d\right)=h_{0}\left(x_{1}, x_{2}\right)$ and $\tilde{f}$ is defined from $f$ likewise. From (32),

$$
\alpha=\boldsymbol{n} \cdot \boldsymbol{e}_{3}=\frac{1}{\sqrt{a^{2}+b^{2}+1}},
$$

thus $\alpha=\frac{1}{\sigma}$. Since $\Delta_{\Gamma_{\boldsymbol{m}_{0}}} \tilde{f}=0$, (50) is equivalent to writing that $\int_{\Gamma_{\boldsymbol{m}_{0}}} \tilde{f} \nabla_{\Gamma_{\boldsymbol{m}_{0}}} \tilde{h}_{0} \cdot \nabla_{\Gamma_{\boldsymbol{m}_{0}}} \varphi=0$, for all $\varphi$ in $H_{0}^{1}\left(\Gamma_{\boldsymbol{m}_{0}}\right)$. Just as in the case of variational problem (36), we can argue from there that $\tilde{h_{0}}$ must be zero: this contradicts our assumption on $h_{0}$ thus (41) cannot hold. Therefore, for every $\boldsymbol{m}_{0}$ in $B$, there is a positive constant $C_{\boldsymbol{m}_{0}}$ such that for all $\boldsymbol{m}$ in $B$ and all $\boldsymbol{h}$ in $H_{0}^{1}(R)$,

$$
\left\|A_{\boldsymbol{m}_{0}} h_{0}-A_{\boldsymbol{m}} h_{m}\right\|_{L^{2}(V)} \geq C_{\boldsymbol{m}_{0}}\left|\boldsymbol{m}_{0}-\boldsymbol{m}\right|
$$


Can the constant $C_{\boldsymbol{m}_{0}}$ in (51) be chosen uniformly in $h_{0}$ and $\boldsymbol{m}_{0}$, or at least uniformly for $h_{0}$ in a bounded set? The following theorem addresses this question. Since the injective operator $A_{m_{0}}$ is compact, its inverse is unbounded, so a non-zero lower bound for $\left\|A_{m_{0}} h_{0}\right\|_{L^{2}(V)}$ has to be considered rather than a lower bound for $\left\|h_{0}\right\|_{H_{0}^{1}(R)}$.

Theorem 3.3. Let $B$ be a closed and bounded subset of geometry parameters $\boldsymbol{m}$ in $\mathbb{R}^{3}$ such that the distance condition (21) holds. Let $A_{m}$ be the crack to boundary operator defined by (22). Let $M_{1}$ and $M_{2}$ be two positive constants. There is a positive constant $C$ such that for all $\boldsymbol{m}, \boldsymbol{m}^{\prime}$ in $B$, all $g$ in $H_{0}^{1}(R)$, and all $h$ in the set

$$
S=\left\{\varphi \in H_{0}^{1}(R):\|\varphi\|_{H_{0}^{1}(R)} \leq M_{2} \text { and } M_{1} \leq\left\|A_{\boldsymbol{m}} \varphi\right\|_{L^{2}(V)} \text { for all } \boldsymbol{m} \in B\right\},
$$

the following estimate holds,

$$
\left\|A_{\boldsymbol{m}} g-A_{\boldsymbol{m}^{\prime}} h\right\|_{L^{2}(V)} \geq C\left|\boldsymbol{m}-\boldsymbol{m}^{\prime}\right| .
$$

Proof: Arguing by contradiction assume that there are sequences $\boldsymbol{m}_{n}$ and $\boldsymbol{m}_{n}^{\prime}$ in $B, g_{n}$ in $H_{0}^{1}(R)$ and $h_{n}$ in $S$ such that $\boldsymbol{m}_{n} \neq \boldsymbol{m}_{n}^{\prime}$ and

$$
\lim _{n \rightarrow \infty} \frac{\left\|A_{\boldsymbol{m}_{n}} g_{n}-A_{\boldsymbol{m}_{n}^{\prime}} h_{n}\right\|_{L^{2}(V)}}{\left|\boldsymbol{m}_{n}-\boldsymbol{m}_{n}^{\prime}\right|}=0 .
$$

By compactness, we may assume that $\boldsymbol{m}_{n}$ converges to some $\tilde{\boldsymbol{m}}$ in $B, \boldsymbol{m}_{n}^{\prime}$ converges to some $\tilde{\tilde{\boldsymbol{m}}}$ in $B$, and $h_{n}$ converges weakly to some $\tilde{h}$ in $H_{0}^{1}(R)$. Note that $A_{\boldsymbol{m}_{n}}$ converges to $A_{\tilde{m}}$ in operator norm, $A_{\boldsymbol{m}_{n}^{\prime}}$ converges to $A_{\tilde{\tilde{m}}}$ in operator norm, and $A_{\boldsymbol{m}_{n}^{\prime}} h_{n}$ converges strongly to $A_{\tilde{\tilde{m}}} \tilde{h}$ in $L^{2}(V)$. As $h_{n}$ is in $S, M_{1} \leq\left\|A_{\boldsymbol{m}_{n}^{\prime}} h_{n}\right\|_{L^{2}(V)}$, so at the limit we have that $\tilde{h} \neq 0$. Assume that $\tilde{\boldsymbol{m}} \neq \tilde{\tilde{\boldsymbol{m}}}$. As,

$$
\left\|\left(I-P_{\boldsymbol{m}_{n}}\right) A_{\boldsymbol{m}_{n}^{\prime}} h_{n}\right\|_{L^{2}(V)} \leq\left\|A_{\boldsymbol{m}_{n}} g_{n}-A_{\boldsymbol{m}_{n}^{\prime}} h_{n}\right\|_{L^{2}(V)},
$$

where as earlier $P_{\boldsymbol{m}_{n}}$ is the orthogonal projection onto $\overline{\mathcal{R}\left(A_{\boldsymbol{m}_{n}}\right)},\left(I-P_{\boldsymbol{m}_{n}}\right) A_{\boldsymbol{m}_{n}^{\prime}} h_{n}$ is also convergent to zero and at the limit we find $A_{\tilde{\tilde{m}} \tilde{h}} \tilde{h}=P_{\tilde{\boldsymbol{m}}} A_{\tilde{\tilde{m}}} \tilde{h}$, so there is an $h^{\prime}$ in $H_{0}^{1}(R)$ such that $A_{\tilde{\tilde{\boldsymbol{m}}}} \tilde{h}=A_{\tilde{\boldsymbol{m}}} h^{\prime}$. By uniqueness Theorem 2.2, we infer that $\tilde{h}=0$ : contradiction. We now know that $\tilde{\boldsymbol{m}}=\tilde{\tilde{\boldsymbol{m}}}$. We first observe that,

$$
\lim _{n \rightarrow \infty} \frac{\left\|\left(I-P_{\boldsymbol{m}_{n}}\right)\left(A_{\boldsymbol{m}_{n}}-A_{\boldsymbol{m}_{n}^{\prime}}\right) h_{n}\right\|_{L^{2}(V)}}{\left|\boldsymbol{m}_{n}-\boldsymbol{m}_{n}^{\prime}\right|}=0 .
$$

Since $\frac{\boldsymbol{m}_{n}-\boldsymbol{m}_{n}^{\prime}}{\left|\boldsymbol{m}_{n}-\boldsymbol{m}_{n}^{\prime}\right|}$ is a sequence on the unit sphere of $\mathbb{R}^{3}$, after possibly extracting a subsequence we may assume that it converges to some $\boldsymbol{q}$ with $|\boldsymbol{q}|=1$. Then as,

$$
\begin{gathered}
A_{\boldsymbol{m}_{n}}=A_{\tilde{\boldsymbol{m}}}+\left(\boldsymbol{m}_{n}-\tilde{\boldsymbol{m}}\right) \nabla A_{\tilde{\boldsymbol{m}}}+\left|\boldsymbol{m}_{n}-\tilde{\boldsymbol{m}}\right| u_{n}, \\
A_{\boldsymbol{m}_{n}^{\prime}}=A_{\tilde{\boldsymbol{m}}}+\left(\boldsymbol{m}_{n}^{\prime}-\tilde{\boldsymbol{m}}\right) \nabla A_{\tilde{\boldsymbol{m}}}+\left|\boldsymbol{m}_{n}^{\prime}-\tilde{\boldsymbol{m}}\right| v_{n},
\end{gathered}
$$

where $\lim _{n \rightarrow \infty} u_{n}=\lim _{n \rightarrow \infty} v_{n}=0$ in operator norm, we infer that $\frac{A_{\boldsymbol{m}_{n}}-A_{\boldsymbol{m}_{n}^{\prime}}}{\left|\boldsymbol{m}_{n}-\boldsymbol{m}_{n}^{\prime}\right|}$ converges to $\partial_{\boldsymbol{q}} A_{\tilde{m}}$ in operator norm. Since $h_{n}$ is weakly convergent to $\tilde{h}$ and the operator $\partial_{\boldsymbol{q}} A_{\tilde{\boldsymbol{m}}}$ is compact, we obtain from the limit (55) the relation $\left(I-P_{\tilde{m}}\right) \partial_{\boldsymbol{q}} A_{\tilde{m}} \tilde{h}=0$. This is akin to relation (47) in the proof of Theorem 3.2. The same argument as in that proof will show that $\tilde{h}=0$ : contradiction. 


\section{Conclusion and perspectives for future work}

We have shown in this paper a uniform stability result for a planar crack inverse problem in half space governed by the Laplace equation. We proved that reconstructing the plane containing the crack is Lipschitz stable despite the fact that the forcing term for the underlying PDE is unknown. This stability result holds under the assumption that the forcing term $g$ is bounded above in $H_{0}^{1}$ norm and the Dirichlet data on the top boundary is bounded below in $L^{2}$ norm.

In future work, we are planning to investigate computational aspects of solving this crack inverse problem. In the case of the full linear elasticity problem, this was done in [14] and in [13, where special random walk techniques were designed and implemented. Our research has shown [13] that finding an objective and automatic way of dealing with regularization parameters is possible thanks to random walks. Conducting a similar study for the case governed by the Laplace equation would present the advantage that the related Green function is orders of magnitude simpler to compute. It would therefore be easier to focus on the development of novel reconstruction algorithms for the posterior probability distribution functions of geometry parameters defining the crack, unhindered by the cost of computing the Green function.

\section{Acknowledgments}

This work was supported by Simons Foundation Collaboration Grant [351025].

\section{A A counter example for the unique solvability of the crack inverse problem in case of Lipschitz cracks}

Define the open disk in $\mathbb{R}^{2}$,

$$
R=\left\{\left(x_{1}, x_{2}\right) \in \mathbb{R}^{2}: \sqrt{x_{1}^{2}+x_{2}^{2}}<2\right\} .
$$

Define two Lipschitz functions from $R$ to $[-1-\sqrt{2},-3+\sqrt{2}]$,

$$
\begin{aligned}
& \psi_{1}\left(x_{1}, x_{2}\right)= \begin{cases}-2, & \text { if } 1 \leq \sqrt{x_{1}^{2}+x_{2}^{2}}<2 \\
-3+\sqrt{2-x_{1}^{2}-x_{2}^{2}}, & \text { if } 0 \leq \sqrt{x_{1}^{2}+x_{2}^{2}}<1,\end{cases} \\
& \psi_{2}\left(x_{1}, x_{2}\right)= \begin{cases}-2, & \text { if } 1 \leq \sqrt{x_{1}^{2}+x_{2}^{2}}<2 \\
-1-\sqrt{2-x_{1}^{2}-x_{2}^{2}}, & \text { if } 0 \leq \sqrt{x_{1}^{2}+x_{2}^{2}}<1 .\end{cases}
\end{aligned}
$$

Cross sections of the graphs of $\psi_{1}$ and $\psi_{2}$ are sketched in Figure1. Next, define the function $g$ in $R$

$$
g\left(x_{1}, x_{2}\right)= \begin{cases}4-x_{1}^{2}-x_{2}^{2}, & \text { if } 1 \leq \sqrt{x_{1}^{2}+x_{2}^{2}}<2 \\ 3, & \text { if } 0 \leq \sqrt{x_{1}^{2}+x_{2}^{2}}<1\end{cases}
$$


$g$ can be extended by zero outside $R$ to obtain a function in $H_{0}^{1}\left(\mathbb{R}^{2}\right)$. For $i=1,2$, let $\Gamma_{i}$ be the open surface defined by the graph of $\psi_{i}$ and define $u^{i}$ be the function on $\mathbb{R}^{3-} \backslash \overline{\Gamma_{i}}$

$$
u^{i}(\boldsymbol{x})=\int_{R} H\left(x_{1}, x_{2}, x_{3}, y_{1}, y_{2}, \psi_{i}\left(y_{1}, y_{2}\right), \boldsymbol{n}_{i}\right) g\left(y_{1}, y_{2}\right) \sigma_{i} d y_{1} d y_{2},
$$

where $H$ is as in (12), $\sigma_{i}$ is defined almost everywhere on $R$ and equals $\sqrt{1+\left(\partial_{x_{1}} \psi_{i}\right)^{2}+\left(\partial_{x_{2}} \psi_{i}\right)^{2}}$, and $\boldsymbol{n}_{i}=\left(-\partial_{x_{1}} \psi_{i},-\partial_{x_{2}} \psi_{i}, 1\right) / \sigma_{i}$, almost everywhere. Let $u=u^{1}-u^{2}$ and $D$, sketched in Figure 1, be the Lipshitz domain defined by

$$
-1-\sqrt{2-x_{1}^{2}-x_{2}^{2}}<x_{3}<-3+\sqrt{2-x_{1}^{2}-x_{2}^{2}}, \quad \sqrt{x_{1}^{2}+x_{2}^{2}}<1 .
$$

Let $u=u^{1}-u^{2}$. We observe that the exterior normal unit vector $\boldsymbol{n}$ to $\partial D$ is $\boldsymbol{n}^{1}$ on $\partial D \cap \Gamma_{1}$

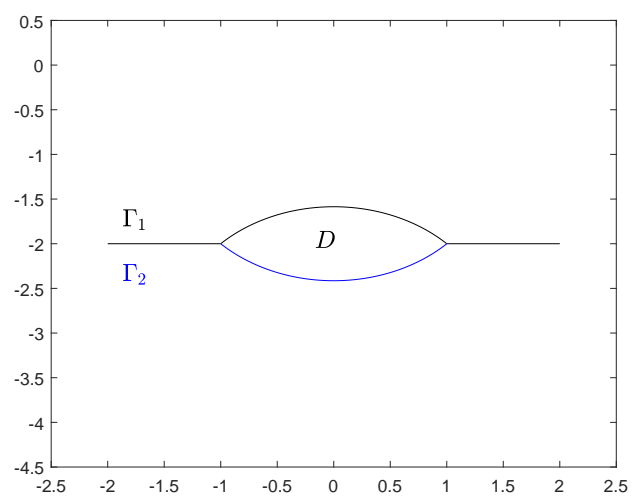

Figure 1: A cross section showing the two open surfaces $\Gamma_{1}$ and $\Gamma_{2}$ together with the domain $D$.

and $-\boldsymbol{n}^{2}$ on $\partial D \cap \Gamma_{2}$, so it follows that,

$$
u(\boldsymbol{x})=3 \int_{\partial D} H(\boldsymbol{x}, \boldsymbol{y}, \boldsymbol{n}) d \sigma(\boldsymbol{y})
$$

for $\boldsymbol{x}$ in $\mathbb{R}^{3-} \backslash \partial D$. By Green's theorem, $u(\boldsymbol{x})=0$ if $\boldsymbol{x} \in \mathbb{R}^{3-} \backslash \bar{D}, u(\boldsymbol{x})=-3$ if $\boldsymbol{x} \in D$. We conclude that for all $\left(x_{1}, x_{2}\right)$ in $\mathbb{R}^{2}, u^{1}\left(x_{1}, x_{2}, 0\right)=u^{2}\left(x_{1}, x_{2}, 0\right)$ and $\partial_{x_{3}} u^{1}\left(x_{1}, x_{2}, 0\right)=$ $\partial_{x_{3}} u^{2}\left(x_{1}, x_{2}, 0\right)$, thus the crack inverse problem is not uniquely solvable without additional assumptions.

\section{B The geometry of $U_{j}$ for $j \geq 2$}

As $\psi_{i}: R_{i} \rightarrow \mathbb{R}$ is Lipschitz continuous, we can extend $\psi_{i}$ as a Lipschitz continuous function from $\overline{R_{i}}$ to $\mathbb{R}, i=1,2$.

Lemma B.1. Let $j \geq 2$ and $\left(x_{1}, x_{2}, x_{3}\right)$ be in $U_{j}$. Then $\left(x_{1}, x_{2}\right) \in R_{1} \cap R_{2}$ and $\psi_{1}\left(x_{1}, x_{2}\right) \neq$ $\psi_{2}\left(x_{1}, x_{2}\right)$. 
Proof: Arguing by contradiction assume that $j \geq 2,\left(x_{1}, x_{2}, x_{3}\right) \in U_{j}$, and $\left(x_{1}, x_{2}\right) \notin R_{1}$. If $\left(x_{1}, x_{2}\right) \notin R_{2}$ then the half line $\left\{\left(x_{1}, x_{2}, t x_{3}\right): t>0\right\}$ is in $U$, so $\left(x_{1}, x_{2}, x_{3}\right) \in U_{1}$ : contradiction.

It follows that $\left(x_{1}, x_{2}\right) \in R_{2}$. If $\psi_{2}\left(x_{1}, x_{2}\right) \geq x_{3}$ then the half-line $\left\{\left(x_{1}, x_{2}, t x_{3}\right): t>1\right\}$ is in $U_{1}$. As $U_{j}$ is open, for some $\alpha>1,\left(x_{1}, x_{2}, \alpha x_{3}\right) \in U_{j}$ : contradiction. If $\psi_{2}\left(x_{1}, x_{2}\right)<x_{3}$, then the line segment $\left\{\left(x_{1}, x_{2}, t x_{3}\right): t \in(0,1]\right\}$ is in $U_{1}$ : contradiction. At this stage we conclude that $\left(x_{1}, x_{2}\right) \in R_{1}$ and similarly $\left(x_{1}, x_{2}\right) \in R_{2}$. There remains to show that $\psi_{1}\left(x_{1}, x_{2}\right) \neq$ $\psi_{2}\left(x_{1}, x_{2}\right)$. Arguing by contradiction, assume that $\psi_{1}\left(x_{1}, x_{2}\right)=\psi_{2}\left(x_{1}, x_{2}\right)$. Then the halfline $L_{1}=\left\{\left(x_{1}, x_{2}, t \psi_{1}\left(x_{1}, x_{2}\right)\right): t>1\right\}$ and the line segment $L_{2}=\left\{\left(x_{1}, x_{2}, t \psi_{1}\left(x_{1}, x_{2}\right)\right)\right.$ : $t \in(0,1)\}$ are in $U_{1}$. Since $j \geq 2$ and $\left(x_{1}, x_{2}, x_{3}\right) \in U_{j},\left(x_{1}, x_{2}, x_{3}\right)$ is neither in $L_{1}$ nor in $L_{2}$. It follows that $x_{3}=\psi_{1}\left(x_{1}, x_{2}\right)$. But as $U_{j}$ is open $\left(x_{1}, x_{2}, \alpha x_{3}\right) \in U_{j}$ for some $\alpha>0$ : contradiction.

Lemma B.2. Fix $j \geq 2$. Then for all $\left(x_{1}, x_{2}, x_{3}\right)$ in $U_{j}, \psi_{1}\left(x_{1}, x_{2}\right)<\psi_{2}\left(x_{1}, x_{2}\right)$, or for all $\left(x_{1}, x_{2}, x_{3}\right)$ in $U_{j}, \psi_{1}\left(x_{1}, x_{2}\right)>\psi_{2}\left(x_{1}, x_{2}\right)$

Proof: The function $\phi_{j}: U_{j} \rightarrow \mathbb{R}, \phi_{j}\left(x_{1}, x_{2}, x_{3}\right)=\psi_{1}\left(x_{1}, x_{2}\right)-\psi_{2}\left(x_{1}, x_{2}\right)$, is continuous. As $U_{j}$ is connected, $\phi_{j}\left(U_{j}\right)$ is connected in $\mathbb{R}$. By Lemma B.1, $0 \notin \phi_{j}\left(U_{j}\right)$.

Lemma B.3. Let $j \geq 2$ and $\Omega_{j}=\left\{\left(x_{1}, x_{2}\right) \in \mathbb{R}^{2}:\left(x_{1}, x_{2}, x_{3}\right) \in U_{j}\right\}$. Then $\Omega_{j}$ is a bounded and connected open subset of $\mathbb{R}^{2}$ and for all $\left(x_{1}, x_{2}\right)$ in $\partial \Omega_{j}, \psi_{1}\left(x_{1}, x_{2}\right)=\psi_{2}\left(x_{1}, x_{2}\right)$.

Proof: The first assertion is clear since $U_{j}$ is open, bounded, and connected in $\mathbb{R}^{3}$. Arguing by contradiction, assume that for some $\left(x_{1}, x_{2}\right)$ in $\partial \Omega_{j}, \psi_{1}\left(x_{1}, x_{2}\right) \neq \psi_{2}\left(x_{1}, x_{2}\right)$. Without loss of generality we may assume that $\psi_{1}\left(x_{1}, x_{2}\right)<\psi_{2}\left(x_{1}, x_{2}\right)$. Since $R_{1} \cap R_{2}$ is open, $\left(x_{1}, x_{2}\right)$ is in $R_{1} \cap R_{2}$, or it is on its boundary, $\partial\left(R_{1} \cap R_{2}\right)$. In the first case, by continuity, there is a positive $\epsilon$ and a positive $\alpha$ such that if $\left(y_{1}, y_{2}\right)$ is in $\left(x_{1}-\alpha, x_{1}+\alpha\right) \times\left(x_{2}-\alpha, x_{2}+\alpha\right) \subset\left(R_{1} \cap R_{2}\right)$, $\psi_{1}\left(y_{1}, y_{2}\right)<\psi_{2}\left(y_{1}, y_{2}\right)+\epsilon$. But then for any element $\left(z_{1}, z_{2}, z_{3}\right)$ in $\left(x_{1}-\alpha, x_{1}+\alpha\right) \times\left(x_{2}-\right.$ $\left.\alpha, x_{2}+\alpha\right) \times\left(\psi_{1}\left(x_{1}, x_{2}\right), \psi_{2}\left(x_{1}, x_{2}\right)\right)$, the line segment from $\left(z_{1}, z_{2}, z_{3}\right)$ to $\left(x_{1}, x_{2}, \psi_{1}\left(x_{1}, x_{2}\right)+\frac{\epsilon}{2}\right)$ is included in $U$, thus the whole set $\left(x_{1}-\alpha, x_{1}+\alpha\right) \times\left(x_{2}-\alpha, x_{2}+\alpha\right) \times\left(\psi_{1}\left(x_{1}, x_{2}\right), \psi_{2}\left(x_{1}, x_{2}\right)\right)$ is in the same connected component $U_{j}$ : this contradicts that $\left(x_{1}, x_{2}\right)$ is in $\partial \Omega_{j}$.

In the second case, $\left(x_{1}, x_{2}\right)$ is in $\partial\left(R_{1} \cap R_{2}\right)$. Without loss of generality say that it is in $\partial R_{1}$. By a continuity argument, a small open ball centered at $\left(x_{1}, x_{2}, \psi_{1}\left(x_{1}, x_{2}\right)+\frac{\epsilon}{2}\right)$ for some small $\epsilon>0$ is included in $U_{1}$ and intersects $U_{j}$, which is a contradiction.

Using lemmas B.1, B.2, and B.3, we see that $U_{j}$ is simple in the $x_{3}$ direction and that for any integrable function $f$ in $U_{j}$,

$$
\int_{U_{j}} f=\int_{\Omega_{j}} \int_{\psi_{k}\left(x_{1}, x_{2}\right)}^{\psi_{l}\left(x_{1}, x_{2}\right)} f\left(x_{1}, x_{2}, x_{3}\right) d x_{3} d x_{1} d x_{2},
$$

where depending on $j \geq 2,(k, l)=(1,2)$ or $(k, l)=(2,1)$.

\section{Proof of Lemma 2.1}

We first state and prove two intermediary results. 
Lemma C.1. Let $\Gamma$ be an open surface in $\mathbb{R}^{3}$ included in the plane $x_{3}=0$ and let $g$ be in $C_{c}^{\infty}(\Gamma)$. Using the notation for $\boldsymbol{x}=\left(x_{1}, x_{2}, 0\right)$ in $\Gamma$,

$$
[v(\boldsymbol{x})]=\lim _{\epsilon \rightarrow 0^{+}} v\left(x_{1}, x_{2}, \epsilon\right)-v\left(x_{1}, x_{2},-\epsilon\right),
$$

the following jump formulas across $\Gamma$ hold for $i=1,2$,

$$
\begin{gathered}
{\left[\int_{\Gamma} G\left(\boldsymbol{x}, \boldsymbol{y}, \boldsymbol{e}_{3}\right) g(\boldsymbol{y}) d \boldsymbol{y}\right]=g(\boldsymbol{x}),} \\
{\left[\int_{\Gamma}\left(\partial_{y_{i}} G\right)\left(\boldsymbol{x}, \boldsymbol{y}, \boldsymbol{e}_{3}\right) g(\boldsymbol{y}) d \boldsymbol{y}\right]=-\partial_{x_{i}} g(\boldsymbol{x}),} \\
{\left[\int_{\Gamma} G\left(\boldsymbol{x}, \boldsymbol{y}, \boldsymbol{e}_{i}\right) g(\boldsymbol{y}) d \boldsymbol{y}\right]=0,} \\
{\left[\int_{\Gamma}\left(\partial_{y_{3}} G\right)\left(\boldsymbol{x}, \boldsymbol{y}, \boldsymbol{e}_{3}\right) g(\boldsymbol{y}) d \boldsymbol{y}\right]=0,} \\
{\left[\partial_{x_{3}} \int_{\Gamma} G\left(\boldsymbol{x}, \boldsymbol{y}, \boldsymbol{e}_{3}\right) g(\boldsymbol{y}) d \boldsymbol{y}\right]=0,} \\
{\left[\partial_{x_{3}} \int_{\Gamma} G\left(\boldsymbol{x}, \boldsymbol{y}, \boldsymbol{e}_{i}\right) g(\boldsymbol{y}) d \boldsymbol{y}\right]=\partial_{x_{i}} g(\boldsymbol{x}),} \\
{\left[\partial_{x_{3}} \int_{\Gamma}\left(\partial_{y_{3}} G\right)\left(\boldsymbol{x}, \boldsymbol{y}, \boldsymbol{e}_{3}\right) g(\boldsymbol{y}) d \boldsymbol{y}\right]=\Delta g(\boldsymbol{x}) .}
\end{gathered}
$$

Proof: We can find a domain $D$ of class $C^{2}$ such that $\Gamma \subset \partial D$ and the exterior normal vector to $\partial D$ is equal to $\boldsymbol{e}_{3}$ on $\Gamma$. Extend $g$ by zero on $\partial D \backslash \Gamma$ to obtain a function in $C^{2}(\partial D)$. Now, given the definition of $G(11),(57)$ is just the classical jump formula for the double layer potential. To show (58), we note that for $x_{3} \neq 0, \boldsymbol{y}$ in $\Gamma$, and $i=1,2$,

$$
\int_{\Gamma}\left(\partial_{y_{i}} G\right)\left(\boldsymbol{x}, \boldsymbol{y}, \boldsymbol{e}_{3}\right) g(\boldsymbol{y}) d \boldsymbol{y}=-\int_{\Gamma} G\left(\boldsymbol{x}, \boldsymbol{y}, \boldsymbol{e}_{3}\right) \partial_{i} g(\boldsymbol{y}) d \boldsymbol{y},
$$

and we just apply (57). Similarly, to show (59), we note that for $x_{3} \neq 0, \boldsymbol{y}$ in $\Gamma$, and $i=1,2$,

$$
\int_{\Gamma} G\left(\boldsymbol{x}, \boldsymbol{y}, \boldsymbol{e}_{i}\right) g(\boldsymbol{y}) d \boldsymbol{y}=-\int_{\Gamma} \Phi(\boldsymbol{x}, \boldsymbol{y}) \partial_{i} g(\boldsymbol{y}) d \boldsymbol{y},
$$

and we use the well known continuity of the single layer potential.

Identity (61) is due to the well known continuity property of the normal derivative of the double layer potential.

To show (60) and (62), we note that for $x_{3} \neq 0$ and $\boldsymbol{y}$ in $\Gamma$, since $\Phi$ depends on $\boldsymbol{x}$ and $\boldsymbol{y}$ only through $\boldsymbol{x}-\boldsymbol{y}$, for $i=1,2$ or 3 ,

$$
\partial_{x_{3}} G\left(\boldsymbol{x}, \boldsymbol{y}, \boldsymbol{e}_{i}\right)=\partial_{x_{3}}\left(\nabla_{\boldsymbol{y}} \Phi(\boldsymbol{x}, \boldsymbol{y}) \cdot \boldsymbol{e}_{i}\right)=-\nabla_{\boldsymbol{y}}\left(\partial_{y_{3}} \Phi(\boldsymbol{x}, \boldsymbol{y})\right) \cdot \boldsymbol{e}_{i}
$$


If $i=3,60$ is now clear due to 61 . To show 62 , for $i=1,2$,

$$
\partial_{x_{3}} \int_{\Gamma} G\left(\boldsymbol{x}, \boldsymbol{y}, \boldsymbol{e}_{i}\right) g(\boldsymbol{y}) d \boldsymbol{y}=\int_{\Gamma}\left(\partial_{y_{3}} \Phi(\boldsymbol{x}, \boldsymbol{y})\right) \partial_{y_{i}} g(\boldsymbol{y}) d \boldsymbol{y}=\int_{\Gamma} G\left(\boldsymbol{x}, \boldsymbol{y}, \boldsymbol{e}_{3}\right) \partial_{y_{i}} g(\boldsymbol{y}) d \boldsymbol{y},
$$

and we can apply (57). To show $(63)$, we use again that $\Phi$ depends on $\boldsymbol{x}$ and $\boldsymbol{y}$ only through $\boldsymbol{x}-\boldsymbol{y}$, so if $x_{3} \neq 0$ and $\boldsymbol{y}$ is in $\Gamma$,

$$
\partial_{x_{3}} \partial_{y_{3}} G\left(\boldsymbol{x}, \boldsymbol{y}, \boldsymbol{e}_{3}\right)=-\partial_{y_{3}}^{2} G\left(\boldsymbol{x}, \boldsymbol{y}, \boldsymbol{e}_{3}\right)=\left(\partial_{y_{1}}^{2}+\partial_{y_{2}}^{2}\right) G\left(\boldsymbol{x}, \boldsymbol{y}, \boldsymbol{e}_{3}\right),
$$

where we have used that $\Delta_{y} \Phi(\boldsymbol{x}, \boldsymbol{y})=0$ if $\boldsymbol{x} \neq \boldsymbol{y}$. Now as $g$ is in $C_{c}^{\infty}(\Gamma)$,

$$
\int_{\Gamma}\left(\partial_{y_{1}}^{2}+\partial_{y_{2}}^{2}\right) G\left(\boldsymbol{x}, \boldsymbol{y}, \boldsymbol{e}_{3}\right) g(\boldsymbol{y}) d \boldsymbol{y}=\int_{\Gamma} G\left(\boldsymbol{x}, \boldsymbol{y}, \boldsymbol{e}_{3}\right)\left(\partial_{y_{1}}^{2}+\partial_{y_{2}}^{2}\right) g(\boldsymbol{y}) d \boldsymbol{y}
$$

and the result follows from (57).

Lemma C.2. Let $\Gamma$ be as in lemma C.1 and $g$ be in $L^{2}(\Gamma)$. Then the jump formulas (57,63) hold in a weak sense, that is for any $\phi$ in $C_{c}^{\infty}(\Gamma)$ and $i=1,2$,

$$
\begin{gathered}
\int_{\Gamma}\left[\int_{\Gamma} G\left(\boldsymbol{x}, \boldsymbol{y}, \boldsymbol{e}_{3}\right) g(\boldsymbol{y}) d \boldsymbol{y}\right] \phi(\boldsymbol{x}) d \boldsymbol{x}=\int_{\Gamma} g(\boldsymbol{x}) \phi(\boldsymbol{x}) d \boldsymbol{x} \\
\int_{\Gamma}\left[\int_{\Gamma}\left(\partial_{y_{i}} G\right)\left(\boldsymbol{x}, \boldsymbol{y}, \boldsymbol{e}_{3}\right) g(\boldsymbol{y}) d \boldsymbol{y}\right] \phi(\boldsymbol{x}) d \boldsymbol{x}=\int_{\Gamma} g(\boldsymbol{x}) \partial_{x_{i}} \phi(\boldsymbol{x}) d \boldsymbol{x}, \\
\int_{\Gamma}\left[\int_{\Gamma} G\left(\boldsymbol{x}, \boldsymbol{y}, \boldsymbol{e}_{i}\right) g(\boldsymbol{y}) d \boldsymbol{y}\right] \phi(\boldsymbol{x}) d \boldsymbol{x}=0, \\
\int_{\Gamma}\left[\int_{\Gamma}\left(\partial_{y_{3}} G\right)\left(\boldsymbol{x}, \boldsymbol{y}, \boldsymbol{e}_{3}\right) d \boldsymbol{y}\right] \phi(\boldsymbol{x}) d \boldsymbol{x}=0, \\
\int_{\Gamma}\left[\partial_{x_{3}} \int_{\Gamma} G\left(\boldsymbol{x}, \boldsymbol{y}, \boldsymbol{e}_{3}\right) d \boldsymbol{y}\right] \phi(\boldsymbol{x}) d \boldsymbol{x}=0, \\
\int_{\Gamma}\left[\partial_{x_{3}} \int_{\Gamma} G\left(\boldsymbol{x}, \boldsymbol{y}, \boldsymbol{e}_{i}\right) d \boldsymbol{y}\right] \phi(\boldsymbol{x}) d \boldsymbol{x}=-\int_{\Gamma} g(\boldsymbol{x}) \partial_{x_{i}} \phi(\boldsymbol{x}) d \boldsymbol{x}, \\
\int_{\Gamma}\left[\partial_{x_{3}} \int_{\Gamma}\left(\partial_{y_{3}} G\right)\left(\boldsymbol{x}, \boldsymbol{y}, \boldsymbol{e}_{3}\right) g(\boldsymbol{y}) d \boldsymbol{y}\right] \phi(\boldsymbol{x}) d \boldsymbol{x}=\int_{\Gamma} g(\boldsymbol{x}) \Delta \phi(\boldsymbol{x}) d \boldsymbol{x} .
\end{gathered}
$$

Proof: Let $\epsilon$ be positive and set,

$$
\boldsymbol{x}^{ \pm}=\left(x_{1}, x_{2}, \pm \epsilon\right), \boldsymbol{y}^{ \pm}=\left(y_{1}, y_{2}, \pm \epsilon\right)
$$

As $\Phi$ depends on $\boldsymbol{x}$ and $\boldsymbol{y}$ only through $\boldsymbol{x}-\boldsymbol{y}$,

$$
\begin{gathered}
G\left(\boldsymbol{x}^{+}, \boldsymbol{y}, \boldsymbol{e}_{3}\right)-G\left(\boldsymbol{x}^{-}, \boldsymbol{y}, \boldsymbol{e}_{3}\right) \\
=\quad \nabla_{\boldsymbol{y}} \Phi\left(\boldsymbol{x}^{+}, \boldsymbol{y}\right) \cdot \boldsymbol{e}_{3}-\nabla_{\boldsymbol{y}} \Phi\left(\boldsymbol{x}^{-}, \boldsymbol{y}\right) \cdot \boldsymbol{e}_{3} \\
=-\nabla_{\boldsymbol{x}} \Phi\left(\boldsymbol{x}^{+}, \boldsymbol{y}\right) \cdot \boldsymbol{e}_{3}+\nabla_{\boldsymbol{x}} \Phi\left(\boldsymbol{x}^{-}, \boldsymbol{y}\right) \cdot \boldsymbol{e}_{3} \\
=-\nabla_{\boldsymbol{x}} \Phi\left(\boldsymbol{x}, \boldsymbol{y}^{-}\right) \cdot \boldsymbol{e}_{3}+\nabla_{\boldsymbol{x}} \Phi\left(\boldsymbol{x}, \boldsymbol{y}^{+}\right) \cdot \boldsymbol{e}_{3},
\end{gathered}
$$


thus by (57),

$$
\lim _{\epsilon \rightarrow 0} \int_{\Gamma}\left(G\left(\boldsymbol{x}^{+}, \boldsymbol{y}, \boldsymbol{e}_{3}\right)-G\left(\boldsymbol{x}^{-}, \boldsymbol{y}, \boldsymbol{e}_{3}\right)\right) \phi(\boldsymbol{x}) d \boldsymbol{x}=\phi(\boldsymbol{y}) .
$$

But the convergence in $(72)$ is known to be uniform for $\boldsymbol{y}$ in $\Gamma$, thus 65 follows.

To prove (66), we observe that

$$
\int_{\Gamma}\left(\partial_{y_{i}} G\right)\left(\boldsymbol{x}^{ \pm}, \boldsymbol{y}, \boldsymbol{e}_{3}\right) \phi(\boldsymbol{x}) d \boldsymbol{x}=-\int_{\Gamma}\left(\partial_{x_{i}} G\right)\left(\boldsymbol{x}^{ \pm}, \boldsymbol{y}, \boldsymbol{e}_{3}\right) \phi(\boldsymbol{x}) d \boldsymbol{x}=\int_{\Gamma} G\left(\boldsymbol{x}^{ \pm}, \boldsymbol{y}, \boldsymbol{e}_{3}\right) \partial_{x_{i}} \phi(\boldsymbol{x}) d \boldsymbol{x}
$$

so the result follows from 65 .

Identities 67, 70 can be derived likewise from lemma C.1. We now show the derivation of (71). As,

$$
\begin{aligned}
& \int_{\Gamma} \partial_{x_{3}} \partial_{y_{3}} G\left(\boldsymbol{x}^{ \pm}, \boldsymbol{y}, \boldsymbol{e}_{3}\right) \phi(\boldsymbol{x}) d \boldsymbol{x}=\int_{\Gamma}-\partial_{x_{3}}^{2} G\left(\boldsymbol{x}^{ \pm}, \boldsymbol{y}, \boldsymbol{e}_{3}\right) \phi(\boldsymbol{x}) d \boldsymbol{x} \\
= & \int_{\Gamma}\left(\partial_{x_{1}}^{2}+\partial_{x_{2}}^{2}\right) G\left(\boldsymbol{x}^{ \pm}, \boldsymbol{y}, \boldsymbol{e}_{3}\right) \phi(\boldsymbol{x}) d \boldsymbol{x}=\int_{\Gamma} G\left(\boldsymbol{x}^{ \pm}, \boldsymbol{y}, \boldsymbol{e}_{3}\right) \Delta \phi(\boldsymbol{x}) d \boldsymbol{x}
\end{aligned}
$$

the result follows from 65 .

To prove Lemma 2.1, it now suffices to extend Lemma C.2 to the case of planar open surfaces that are not necessarily included in the plane $x_{3}=0$. Since the fundamental solution $\Phi$ given by (6) satisfies $\Phi(T \boldsymbol{x}, T \boldsymbol{y})=\Phi(\boldsymbol{x}, \boldsymbol{y})$, for all $\boldsymbol{x}$ and $\boldsymbol{y}$ in $\mathbb{R}^{3}$ such that $\boldsymbol{x} \neq \boldsymbol{y}$ where $T$ is any rotation or translation of $\mathbb{R}^{3}$, we can obtain formulas (13) to (19) by a straightforward generalization of Lemma C.2. Formula (20) can be obtained from (17) by applying Green's formula on $\Gamma$.

\section{References}

[1] Andrea Aspri, Elena Beretta, and Anna L Mazzucato. Dislocations in a layered elastic medium with applications to fault detection. preprint arXiv:2004.00321v1, 2020.

[2] Andrea Aspri, Elena Beretta, Anna L Mazzucato, and V Maarten. Analysis of a model of elastic dislocations in geophysics. Archive for Rational Mechanics and Analysis, 236(1):71-111, 2020.

[3] Martin Costabel. Boundary integral operators on lipschitz domains: elementary results. SIAM Journal on Mathematical Analysis, 19(3):613-626, 1988.

[4] Cristian Dascalu, Ioan R Ionescu, and Michel Campillo. Fault finiteness and initiation of dynamic shear instability. Earth and Planetary Science Letters, 177(3):163-176, 2000.

[5] Zhonghai Ding. A proof of the trace theorem of sobolev spaces on lipschitz domains. Proceedings of the American Mathematical Society, 124(2):591-600, 1996. 
[6] Avner Friedman and Michael Vogelius. Determining cracks by boundary measurements, 1989. http://conservancy.umn.edu/bitstream/handle/11299/4926/476.pdf.

[7] Emilio Gagliardo. Caratterizzazioni delle tracce sulla frontiera relative ad alcune classi di funzioni in $n$ variabili. Rendiconti del seminario matematico della universita di Padova, $27: 284-305,1957$.

[8] Ioan R Ionescu and Darko Volkov. An inverse problem for the recovery of active faults from surface observations. Inverse problems, 22(6):2103, 2006.

[9] Ioan R Ionescu and Darko Volkov. Earth surface effects on active faults: An eigenvalue asymptotic analysis. Journal of Computational and Applied Mathematics, 220(1):143$162,2008$.

[10] Tosio Kato. Perturbation theory for linear operators, volume 132. Springer Science \& Business Media, 2013.

[11] Faouzi Triki and Darko Volkov. Stability estimates for the fault inverse problem. Inverse problems, 35(7), 2019.

[12] D. Volkov, C. Voisin, and Ionescu I.R. Determining fault geometries from surface displacements. Pure and Applied Geophysics, 174(4):1659-1678, 2017.

[13] Darko Volkov. A stochastic approach to mixed linear and nonlinear inverse problems with applications to seismology. preprint arXiv:2007.05347, 2020.

[14] Darko Volkov and Joan Calafell Sandiumenge. A stochastic approach to reconstruction of faults in elastic half space. Inverse Problems \& Imaging, 13(3):479-511, 2019.

[15] Darko Volkov, Christophe Voisin, and Ioan Ionescu. Reconstruction of faults in elastic half space from surface measurements. Inverse Problems, 33(5), 2017. 